\title{
Design for Tolerance of Electro-Mechanical Assemblies: An Integrated Approach
}

\author{
Y. Narahari, R. Sudarsan, K.W. Lyons, M.R. Duffey, and R.D. Sriram
}

Y. Narahari, The George Washington University, Washington, DC 20052 and the National Institute of Standards and Technology, Gaithersburg, MD 20899 (Visiting). Permanent Affiliation: Computer Science and Automation, Indian Institute of Science, Bangalore 560 012, India. Email: hari@csa.iisc.ernet.in

R. Sudarsan, The George Washington University, Washington, DC 20052 and the National Institute of Standards and Technology, Gaithersburg, MD 20899 (Visiting). Email: sudarsan@cme.nist.gov

K.W. Lyons, Engineering Design Technologies Group, Manufacturing Systems Integration Division, National Institute of Standards and Technology, Gaithersburg, MD 20899. Email: klyons@darpa.mil

M.R. Duffey, Department of Engineering Management, The George Washington University, Washington, DC 20052. Email: duffey@seas.gwu.edu

R.D. Sriram, Engineering Design Technologies Group, Manufacturing Systems Integration Division, National Institute of Standards and Technology, Gaithersburg, MD 20899. Email: sriram@cme.nist.gov 


\section{Contents}

1 Introduction $\quad 7$

1.1 Current Status of Design Tolerancing . . . . . . . . . . . . 7

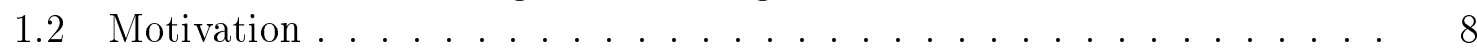

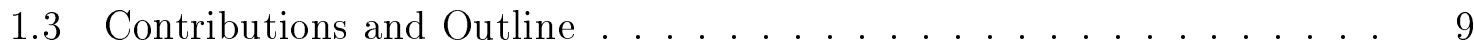

2 Motivating Example $\quad 11$

2.1 A Chassis-like Mechanical Assembly . . . . . . . . . . . . . . 11

2.1.1 Assembly Response Functions . . . . . . . . . . . . . . . . . 11

2.1.2 Design of the Assembly Process . . . . . . . . . . . . . 13

2.2 Need and Potential for an Integrated Approach . . . . . . . . . . 16

3 Assembly Models for Tolerancing $\quad 16$

3.1 Relevant Assembly Models . . . . . . . . . . . . . . . . 17

3.2 Use of Assembly Models for Tolerancing . . . . . . . . . . . . . . 19

3.3 Assembly and Tolerance Representation through the Design Process . . 21

4 Design Tolerancing: Methods and Best Practices 22

4.1 Methods for Tolerance Analysis . . . . . . . . . . . . . . . 24

4.1.1 Linear Propagation . . . . . . . . . . . . . . . . 26

4.1.2 Non-linear Propagation (Extended Taylor Series) . . . . . . . 27

4.1.3 Numerical Integration . . . . . . . . . . . . . . . 27

4.1.4 Monte Carlo Simulation . . . . . . . . . . . . . . 27

4.2 Methods for Tolerance Synthesis . . . . . . . . . . . . . . . 28

4.2.1 Iterative Methods based on Analysis . . . . . . . . . . . . 29

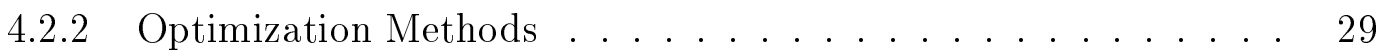

4.2.3 Design of Experiments . . . . . . . . . . . . . . . . . . 30

4.3 Best Practices . . . . . . . . . . . . . . . . 30

4.3.1 Motorola Six Sigma Program . . . . . . . . . . . . 31

4.3.2 Xerox Holistic Probabilistic Design . . . . . . . . . . . 33

4.3.3 Taguchi Methods .................. 35

5 Design for Tolerance Process $\quad 37$

5.1 Design Process for Assemblies . . . . . . . . . . . . . . . 37

5.2 Design Tolerancing: An Incremental Process . . . . . . . . . . . . . . 39

5.3 Design for Tolerance: A Multilevel Approach . . . . . . . . . . . . . . . 40

5.3.1 TR Level 1: Assembly Layout and Configuration . . . . . . . . 41

5.3.2 TR Level 2: Location Logic and Assembly Features . . . . . . . 41

5.3.3 TR Level 3: Assembly Planning and Sequencing . . . . . . . . . 43

5.3.4 TR Level 4: Detailed Tolerance Analysis and Synthesis . . . . . 43

5.4 Design for Tolerance: An Integrated Approach . . . . . . . . . . . 43 
5.4 .1 Design Process . . . . . . . . . . . . . . . . . 44

5.4 .2 Assembly Models . . . . . . . . . . . . . . . . . 44

5.4.3 Tolerance Analysis and Synthesis . . . . . . . . . . . . 44

6 Example 44

6.1 Selecting a Configuration . . . . . . . . . . . . . . . 45

6.2 Selecting Location Logic and Assembly Features . . . . . . . . . . . 47

6.3 Selection of Assembly Sequence . . . . . . . . . . . . . . . . 49

6.4 Detailed Analysis and Synthesis . . . . . . . . . . . . . . . . 49

6.4 .1 Tolerance Analysis . . . . . . . . . . . . . . . . . 49

6.4 .2 Design . . . . . . . . . . . . . . . . 50

7 Discussion and Future Work $\quad 50$

7.1 Implementation of a DFT Environment . . . . . . . . . . . . . 51

7.2 Standards Development . . . . . . . . . . . . . . . 51 


\section{List of Figures}

1 Assembly models, design process stages, and tolerancing tools . . . . 10

2 A mechanical assembly comprising two subassemblies . . . . . . . . . 12

3 Three different configurations of the lower subassembly . . . . . . . . . 14

4 Datum flow chains representing different location logics . . . . . . . . 15

5 A relational representation of the assembly of Figure $2 \ldots \ldots$. . . . 18

6 A hierarchical model of the assembly of Figure 2 . . . . . . . . . 19

7 A datum flow chain for the assembly of Figure 2 . . . . . . . . . . 19

8 Assembly models for tolerancing . . . . . . . . . . . . . . . 23

9 Methods and best practices for tolerancing . . . . . . . . . . . 25

10 Design stages and activities in the SIMA reference architecture. Source: NIST Internal Report 5939 [62] . . . . . . . . . . . . . . . . . . 39

11 An architecture for design for tolerance. Legend: LD - Liaison Diagram; DFC - Datum Flow Chain; HM - Hierarchical Model; OOM Object Oriented Model; AS - Assembly Sequence; PKCs - Product Key Characteristics; AKCs - Assembly Key Characteristics; ARF - Assembly Response Function; PCD - Process Capability Data . . . . . . . . . . 42

12 Three different configurations of the assembly . . . . . . . . . . 46

13 Datum flow chains representing different location logics . . . . . . . 48 


\section{SYMBOLS AND NOTATION}

$\begin{array}{ll}i=1, \ldots, n & \text { Index for individual dimensions } \\ Y & \text { Assembly response characteristic } \\ & \text { (such as assembly gap or any function) } \\ Y_{1}, \ldots, Y_{m} & \text { Individual assembly response characteristics } \\ X_{i} & \text { Dimension or characteristic of an individual part } \\ Y=f\left(X_{1}, \ldots, X_{n}\right) & \text { Assembly response function } \\ \tau & \text { Target value of } Y \\ l(Y) & \text { Loss function (Loss when } Y \text { deviates from } \tau) \\ \mu, \mu_{Y} & \text { Expected value of } Y \\ \mu_{i} & \text { Expected value of } X_{i} \\ \sigma, \sigma_{Y} & \text { Standard deviation of } Y \\ \sigma_{i} & \text { Standard deviation of } X_{i} \\ T & \text { Tolerance of } Y \\ T_{i} & \text { Tolerance of } X_{i} \\ L & \text { Expected quadratic loss, } E[l(Y)] \text { or } \\ U & \text { Lower specification limit of } Y \text { (context sensitive) } \\ C_{p} & \text { Upper specification limit of } Y \\ C_{p k} & \text { Process capability index without mean shift } \\ C_{p i}, C_{p k i} & \text { Process capability index with mean shift } \\ P r\{X\} & \text { Process capability indices for individual parts or processes } \\ \text { Taguchi Methods } & \text { Probability of } X \\ \text { Robust Design } & \text { Registered trademark of American Supplier Institute } \\ \text { ANSI } & \text { Registered trademark of American Supplier Institute } \\ \text { ASME } & \text { American National Standards Institute } \\ & \text { American Society of Mechanical Engineers }\end{array}$




\title{
Design for Tolerance of Electro-Mechanical Assemblies: An Integrated Approach
}

\author{
Y. Narahari, R. Sudarsan, K.W. Lyons, M.R. Duffey, and R.D. Sriram
}

\begin{abstract}
Tolerancing decisions can profoundly impact the quality and cost of electro-mechanical assemblies. Existing approaches to tolerance analysis and synthesis in design entail detailed knowledge of geometry of the assemblies and are mostly applicable during advanced stages of design, leading to a less than optimal design process. During the design process of assemblies, both the assembly structure and associated tolerance information evolve continuously. Therefore, significant gains can be achieved by effectively using this information to influence the design of the assembly. Motivated by this, we identify and explore two goals for future research that we believe can enhance the scope of tolerancing for the entire design process. The first goal is to advance tolerancing decisions to the earliest possible stages of design. This issue raises the need for effective representation of tolerancing information during different stages of design and for effective assembly modeling. The second goal addresses the appropriate, synergistic use of available methods and best practices for tolerance analysis and synthesis, at successive stages of design. Pursuit of these goals leads to the definition of a multi-level approach that enables tolerancing to be addressed at successive stages of design in an incremental fashion. The resulting design process, which we call the design for tolerance process, integrates three important domains: (1) design activities at successive stages of design; (2) assembly models that evolve continuously through the design process; and (3) methods and best practices for tolerance analysis and synthesis. We demonstrate major steps of our proposed approach through a simple, yet illustrative, example.
\end{abstract}

\section{KEYWORDS}

Tolerance Analysis, Tolerance Synthesis, Design Tolerancing, Assembly Design Process, Assembly Modeling, Tolerance Representation, Statistical Tolerancing, System Level Tolerancing. 


\section{Introduction}

Tolerancing is a critical issue in the design of electro-mechanical assemblies. In a recent workshop at the National Institute of Standards and Technology (NIST) [1], several leading researchers from industry, academia, and government emphasized the need for investigating assembly level tolerancing issues and for developing tolerancing standards related to assembly. Tolerancing is a major component in the OpenADE (Open Assembly Design Environment) architecture being developed and implemented at NIST [2]. Tolerancing includes both tolerance analysis and tolerance synthesis. In the context of electro-mechanical assembly design, tolerance analysis refers to evaluating the effect of variations of individual part or subassembly dimensions on designated dimensions or functions of the resulting assembly. Tolerance synthesis refers to allocation of tolerances to individual parts or subassemblies based on tolerance or functional requirements on the assembly. In this paper, we use the phrase design tolerancing to refer to tolerance analysis and synthesis during design.

\subsection{Current Status of Design Tolerancing}

Existing approaches to design tolerancing in electro-mechanical assemblies generally require detailed knowledge of the geometry of the assemblies and are mostly applicable during advanced stages of design. The current industry practice is to assign tolerances only during late stages of design, after nominal dimensions have been fixed by designers. Many firms use Monte Carlo simulation to conduct tolerance analysis on a detailed geometric model of the product. There are some important recent efforts, albeit preliminary, that attempt tolerancing decisions during early stages of design. These include the work based on key characteristics [3,4]; and assembly-oriented design using assembly representations such as datum flow chains [5,6]. Though some important design related decisions can potentially be enabled by these approaches during early stages of design, the actual tolerance analysis would require at least a rough geometric description of the assembled product.

Both worst-case tolerancing and to a lesser extent, statistical tolerancing, are currently practiced in industry [7]. Worst-case tolerancing involves establishing the dimensions and tolerances such that any possible combination will produce a functional assembly, i.e. the probability of non-assembly is identically equal to zero. Consequently, worst-case tolerancing can lead to excessively tight part tolerances and hence high production costs. Statistical tolerancing is a more practical and economical way of looking at tolerances and works on setting the tolerances so as to assure a desired yield, accepting a small percent of non-conformance.

There is now a vast body of literature on tolerance analysis and synthesis, with several survey papers available on important topics [8-19]. There are several software packages available exclusively for tolerance analysis and synthesis [17]. These packages 
are mostly simulation-based; simple analytical or probabilistic techniques are also provided. Industry best practices in design tolerancing include the well-known Motorola six sigma program [20]. Quality engineering techniques such as Taguchi Methods [21] are popular among some industries. There are also proprietary methods and software such as HPD (Holistic Probabilistic Design) from Xerox [22, 23]. Monte Carlo simulation is the most popular technique used by industries and commercial packages.

Dimensional tolerancing has evolved mostly as an industrial practice without strong theoretical foundations [16]. The best tolerancing practices were collected and made available through an evolving series of tolerancing standards [24-27]. All international and most national standards have codified only worst-case tolerancing [7]. There are a few company specific internal standards for statistical tolerancing, such as in IBM [28] and Motorola [20]. The latest ANSI Y14.5M-1994 standard on dimensioning and tolerances $[26,29]$ provides a provision for including statistical tolerances. Currently, mathematically sound definitions of the syntax and semantics of statistical tolerancing are under development for inclusion into standards [27]. An ISO standard for statistical tolerancing is evolving [7].

\subsection{Motivation}

Tolerances must be considered early in the design cycle to develop product specifications for quality assemblies that can be produced cost-effectively. However, as described above, existing approaches to design tolerancing entail detailed knowledge of geometry of the assemblies and are applicable mostly during advanced stages of design, thus leading to a less than optimal design process. During the design process of assemblies, both the assembly structure and associated tolerance information evolve continuously. Therefore, significant gains can be achieved by effectively using this information to influence the design of the assembly. The success of Design for $X$ concepts has established beyond doubt the efficacy of providing feedback on downstream manufacturing concerns. Motivated by this, we identify and explore two goals for future research that we believe can enhance the scope of tolerancing to the entire design process. The first goal is to advance tolerancing decisions to the earliest possible stages of design. This issue raises the need for effective representation of tolerancing information during early stages of design and for effective assembly modeling. These assembly models and tolerance representations should enable the designer to incrementally understand the build-up or propagation of tolerances and optimize the layout, features, or assembly realizations so as to ensure ease of tolerance delivery. The second goal addresses the appropriate, synergistic use of available methods and best practices for tolerance analysis and synthesis, at successive stages of design. Pursuit of these goals leads to the definition of a multi-level approach that enables tolerancing to be addressed at successive stages of design in an incremental, continuous, ongoing fashion. 


\subsection{Contributions and Outline}

The primary contribution of this paper is to propose a multi-level approach to design tolerancing, which we call design for tolerance, to enable tolerancing to be addressed at successive stages of design in an incremental, continuous ongoing fashion. The proposed approach integrates three design-related domains:

- Design activities at successive stages of design

- Assembly models for tolerancing that evolve continuously during the design process

- Methods and best practices for tolerance synthesis and analysis

Figure 1 shows a preview of the three major threads in the proposed methodology. A detailed description of this exhibit appears in the rest of this paper.

The paper is organized as follows. Section 2 presents an example of a chassis-like mechanical assembly and helps motivate the objectives of this paper. Different stages of its design process are delineated, from a tolerancing perspective, to bring out the need for and potential of an integrated, incremental approach to design tolerancing.

In Section 3, we look into existing and emerging assembly modeling approaches that are appropriate to use during different stages of design. The leftmost part of Figure 1 summarizes the assembly models for tolerancing. First, we survey assembly representations based on solid models, relational models, hierarchical models, and datum flow chains. Next, we investigate how these assembly models are useful for design tolerancing at different stages of design. We then identify the requirements and capabilities of an ideal model of assembly for tolerancing that can be used through successive stages of the design process.

Section 4 is devoted to a critical survey of methods and best practices for design tolerancing. (See Figure 1, rightmost part, for a preview of the methods and best practices). First we present important methods for tolerance analysis. These include methods based on: worst-case tolerancing; root sum of squares; extended Taylor series; quadrature techniques; and Monte Carlo simulation. Next, we look into tolerance synthesis methods: methods based on tolerance analysis; methods based on optimization; and methods based on design of experiments. We then outline three industry best practices in design tolerancing: the Motorola six sigma program; the Xerox Holistic Probabilistic Design; and the Taguchi robust design methodology.

In Section 5, we present a four-level, integrated approach for incremental and continuous tolerancing through successive stages of design. First, we establish a broad framework for assembly design process by looking into several candidate viewpoints in the literature. The middle part of Figure 1 shows this multi-level design for tolerance process. Next, we describe the four levels of the design for tolerance process and 


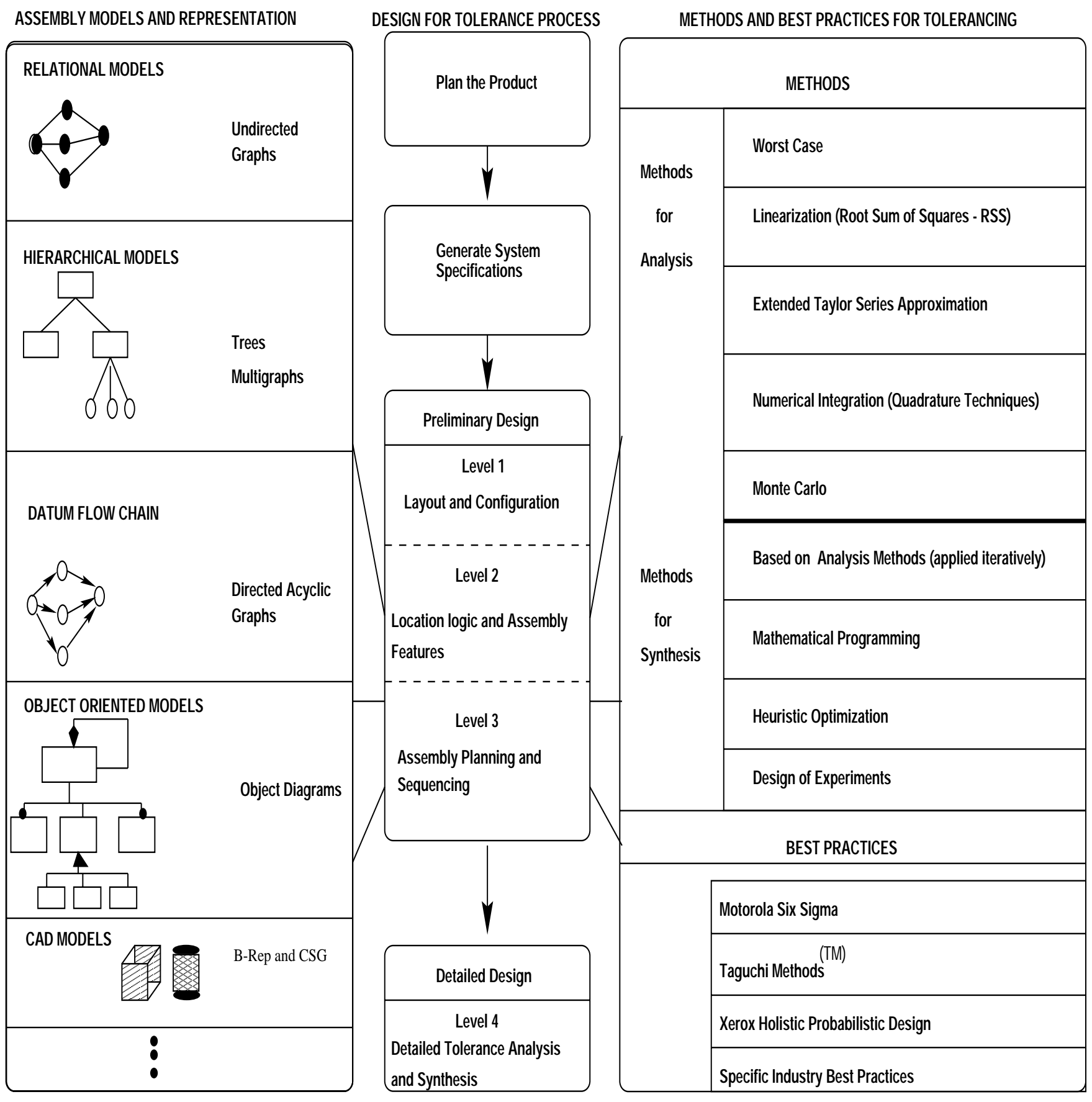

Figure 1: Assembly models, design process stages, and tolerancing tools 
establish the coupling between these levels, the assembly models, and the tolerancing methods and best practices.

In Section 6, we consider a simple, representative example and delineate the major steps of our approach. Section 7 concludes the paper with a statement of what lies ahead and the potential implications of this work.

\section{Motivating Example}

In this section, we present an example of a mechanical assembly. This example is chosen to illustrate the significant potential of using tolerancing considerations at successive stages of design. There are several examples in the literature that describe various ways in which tolerancing considerations can be used during early stages of design. For example, several case study articles in [30] describe tolerance related decisions at different stages of design. In [31], Altschul and Scholz discuss the tolerancing issues that arise when assembling a cargo door to an airplane body. When the cargo door, fitted with several hinges, is assembled to an airplane body, tolerancing problems could result, necessitating a careful tolerance analysis to be done. Problems such as how many hinges to use and how many gaps and lugs to have in a hinge also have tolerancing implications and represent decisions during early stages of design. More recently, Whitney [6], has provided several examples of illustrative assembly scenarios where tolerancing comes to play a decisive role in early stages of design.

Here, we present a simple and illustrative assembly example, give a rough sketch of its design process, and bring out the important role tolerancing considerations can play in successive stages of its design.

\subsection{A Chassis-like Mechanical Assembly}

We consider a simple chassis-like mechanical assembly comprising two major subassemblies - a lower subassembly (main body) and an upper subassembly (cover). (See Figure 2 ). The lower subassembly comprises an envelope $\mathrm{E}$ and three parts $\mathrm{A}, \mathrm{B}$, and $\mathrm{C}$ to be assembled into the envelope. The upper cover is the subassembly D, which is designed to fit into the lower subassembly. Figure 2 is intended to depict only a conceptual view of this assembly; the form shown is not to be viewed as implying any geometry or shape.

\subsubsection{Assembly Response Functions}

Let $l_{a}, l_{b}$, and $l_{c}$ be the lengths of the parts $\mathrm{A}, \mathrm{B}$, and $\mathrm{C}$ respectively; $l_{e}$, the length of the inner boundary of the envelope $E$; and $l_{1}, l_{2}$, the lengths of the left arm and the right arm, respectively, of the cover D. Denote the assembly gap between parts A and B by $g_{a b}$ and define similarly the assembly gaps $g_{b c}, g_{e a}$, and $g_{c e}$. The lengths 


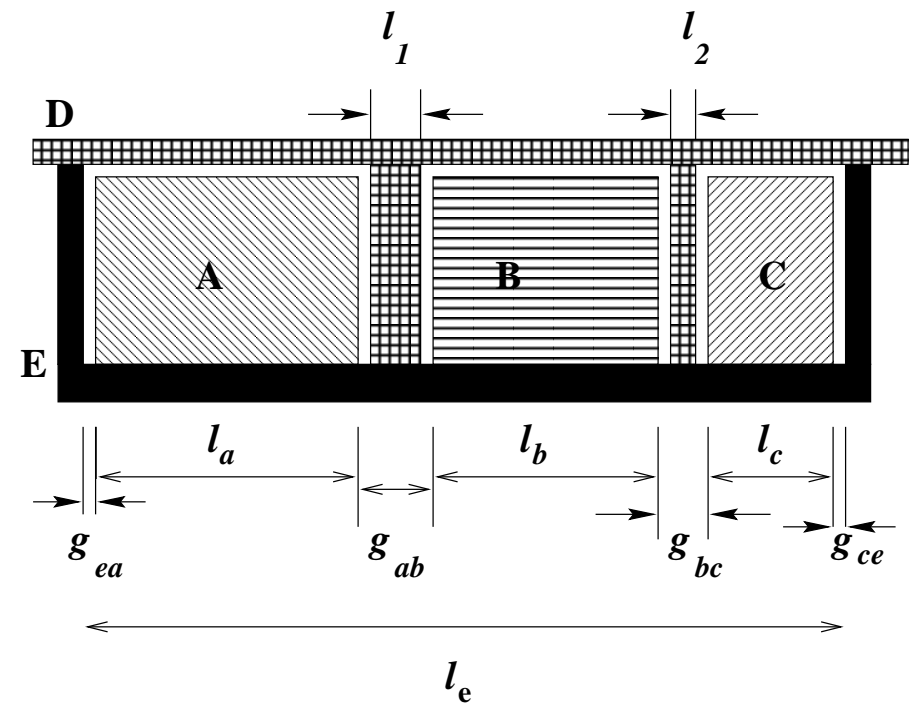

Figure 2: A mechanical assembly comprising two subassemblies

$l_{a}, l_{b}, l_{c}, l_{1}, l_{2}$, and $l_{e}$ can be considered as continuous random variables with some known distributions. Define the random variables $Y_{1}, Y_{2}, Y_{3}$, and $Y_{4}$ as follows:

$$
\begin{aligned}
Y_{1} & =g_{a b}-l_{1} \\
Y_{2} & =g_{b c}-l_{2} \\
Y_{3} & =g_{e a} \\
Y_{4} & =g_{c e}
\end{aligned}
$$

We call these assembly response functions. In the present case, these define the various assembly gaps in the above assembly. The conformance and functionality of the assembly, in this case, are assembly fit criteria:

1. The left arm of the cover D should fit into the gap $g_{a b}$

2. The right arm of $\mathrm{D}$ should fit into the gap $g_{b c}$

3. There should be no interference between $\mathrm{E}$ and $\mathrm{A}$

4. There should be no interference between E and C.

Mathematically, these can be described as:

$$
Y_{i} \geq 0 \text { for } i=1,2,3,4
$$

The conformance criterion in an assembly can be more general than the fit criterion above. In the most general case, we have an assembly response function $Y$ that is an 
arbitrary function of certain input variables $X_{1}, \ldots, X_{n}, Y=f\left(X_{1}, \ldots, X_{n}\right)$, and the conformance or functionality criterion will require $Y$ to lie in a designated tolerance zone. A tolerance zone for a given element (size or feature or form) defines the range of allowable variations of the nominal element. For example, if the length of a part is of interest, then an interval around the nominal length becomes a tolerance zone.

It is important to understand how the design process influences tolerancing issues. Observe that the gaps $g_{a b}, g_{b c}, g_{e a}$, and $g_{c e}$ are decided by the sequence in which we assemble the components and, in general, on the design decisions taken during the design process. At the commencement of design, only the above four relations for $Y_{i}$ $(i=1,2,3,4)$ are known. The exact expressions for the gaps get decided as appropriate design decisions are taken. The order in which the gaps are established is also decided by the design process. For example, if part $\mathrm{A}$ is assembled to the envelope $\mathrm{E}$ first, the gap $g_{a b}$ is established. If part B is next assembled, followed by part C, then gaps $g_{a b}$ and $g_{b c}$ are realized in that order. The gap $g_{c e}$ is then automatically decided by the expression:

$$
g_{c e}=l_{e}-l_{a}-g_{e a}-l_{b}-g_{a b}-l_{c}-g_{b c}
$$

Thus, for the considered sequence of assembly, gaps $g_{e a}, g_{a b}$, and $g_{b c}$ are decided in that order and the gap $g_{c e}$ is dependent on the first three gaps. The first three gaps are decided essentially by the accuracy and process capability of the involved assembly steps or fixturing processes. The order of appearance of the terms in the above equation is important since it reflects the assembly sequence.

Another important issue is the level of detail of an assembly response function. For example, consider the function $Y_{1}=g_{a b}-l_{1}$. $Y_{1}$ only indicates whether the overall dimension $l_{1}$ can fit into the gap $g_{a b}$. At an early stage of design, this requirement may be adequate enough. Later in the design process, however, one may be interested in more details. For example, a left clearance and a right clearance may be specified while assembling the left arm into the gap $g_{a b}$. Thus an assembly response function can evolve through the design process. Tolerance decisions during early design are based on aggregate or approximate versions of the response function. Another related issue is the progression from linear dimensions to complex 3D geometries as design matures. For instance, during early design, we may deal with $l_{1}$ and $g_{a b}$ as linear dimensions, but as the design process unfolds, these variables can assume a nonlinear or 3D form. This again is caused by the evolution in the assembly response function.

\subsubsection{Design of the Assembly Process}

We now give a rough sketch of how the above assembly may be designed from an early conceptual stage and bring out the relevance and potential of tolerance related decisions at different stages of the design process. A more generic description of the design process for electro-mechanical assemblies appears in Section 5. The design will start with planning of the product, conceptualization, and generating the engineering 

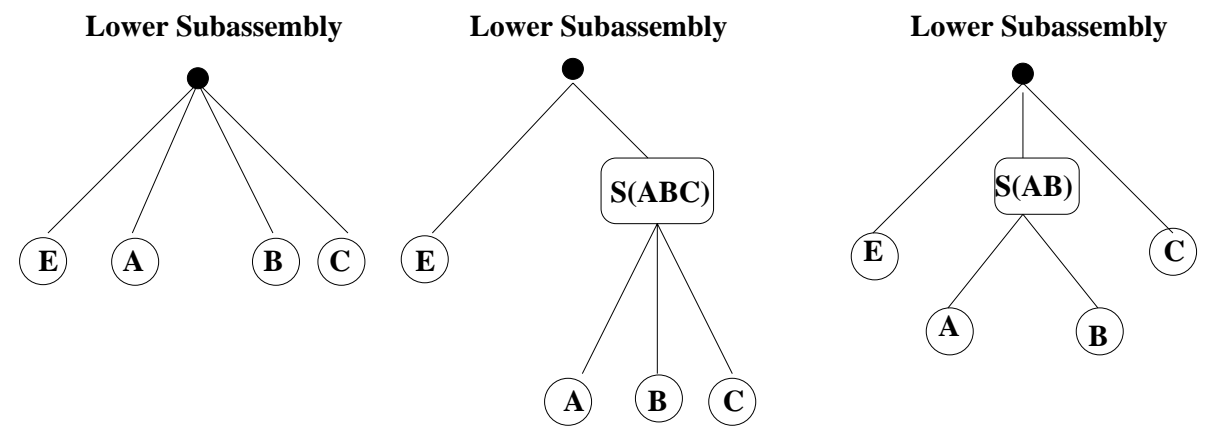

Figure 3: Three different configurations of the lower subassembly

specifications for the parts and the assembly. Since the lower subassembly and the upper subassembly are separate units, their design can proceed separately and in parallel. There is no need for designers to commit to any geometry during these early stages of design. The expressions for the assembly response functions $Y_{1}$ and $Y_{2}$ can be formulated very early in the design process, whereas the expressions for $Y_{3}$ and $Y_{4}$ can only be formulated later, as explained already. However, the assembly criteria $Y_{i} \geq 0$ for $i=1,2,3,4$ are known during early design itself. Note that $Y_{3}$ and $Y_{4}$ are related to the lower subassembly, while $Y_{1}$ and $Y_{2}$ are concerned with the interface between the two subassemblies.

Let us focus on the lower subassembly. We present four levels of decisions with respect to this subassembly, each more downstream than the previous one in the design process.

\section{Selecting a Configuration}

Call the lower subassembly P. Figure 3 shows three possible ways of configuring the four parts A, B, C, and E into P - there could be other configurations as well. In Configuration 1, all four parts are treated as individual parts and the assembly takes place in stages. In Configuration 2, $\mathrm{P}$ is composed of $\mathrm{E}$ and a subassembly that consists of parts A, B, and C. The motivation for considering this latter configuration could be that the subassembly is available off-the-shelf from a known supplier. Likewise, Configuration 3 is another candidate. In this case, the subassembly composed of A and $\mathrm{B}$ might be available from a different supplier. It is clear that the process capabilities and the associated parametric variations of the parts and subassemblies will influence the choice of configuration. The selection of one of the above three configurations could be based on how well the configuration enables proper fitting of the parts inside the envelope. Such decisions certainly need not wait until late in the design process. 


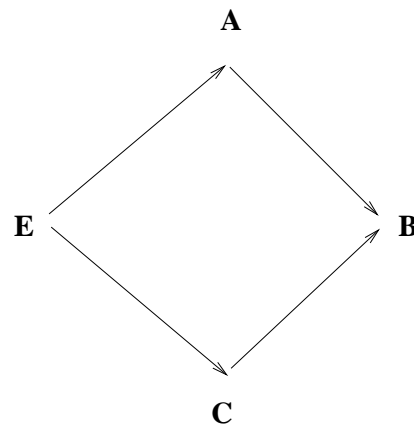

Candidate 1 (B last)

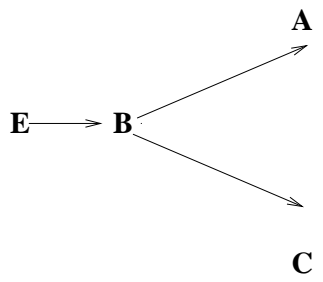

Candidate 2 (B first)

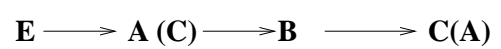

Candidate 3 (B middle)

Figure 4: Datum flow chains representing different location logics

\section{Selecting Location Logic}

In this stage of design, our interest is in deciding the manner in which parts are located with respect to one another (location logic). Figure 4 shows three candidate location logics. Candidate 1 corresponds to a location scheme where $\mathrm{A}$ and $\mathrm{C}$ are first located (in some order) with respect to a datum on the envelope $\mathrm{E}$ and $\mathrm{B}$ is next located relative to $\mathrm{A}$ and $\mathrm{C}$. This scheme can be realized through the assembly sequence $\mathrm{E} \rightarrow$ $\mathrm{A} \rightarrow \mathrm{C} \rightarrow \mathrm{B}$ or the sequence $\mathrm{E} \rightarrow \mathrm{C} \rightarrow \mathrm{A} \rightarrow \mathrm{B}$. In general, a given location logic can be translated into several assembly sequences, thus location logic can be decided earlier than the assembly sequence. Both assembly sequences here are such that component $\mathrm{B}$ is assembled last. In Candidate 2 logic, $\mathrm{B}$ is the first one to be assembled into the envelope, whereas Candidate 3 logic corresponds to those sequences in which $\mathrm{B}$ is assembled in the middle between $\mathrm{A}$ and $\mathrm{C}$ (these two in any order). The directed acyclic graphs in Figure 4 are called datum flow chains [5,6]. They are described in more detail in Section 4. From the conceptual diagram of Figure 2, it is clear that Candidate 1 may necessitate $\mathrm{A}$ and $\mathrm{C}$ to have two mating features; Candidate 2 may entail just one assembly feature each on $\mathrm{A}$ and $\mathrm{C}$; and Candidate 3 may require either $\mathrm{A}$ or $\mathrm{C}$ to have two features while the other may have just one feature. One can evaluate, using simple probabilistic arguments and appropriate process capability data, these candidates based on ease of tolerance delivery. For example, Candidate 3 is likely to be better if there is high uncertainty in the dimension of $\mathrm{B}$. The computation here would involve finding the probability that the assembly response functions $Y_{i}$ lie in the desired tolerance zones. But once a candidate logic is selected, only those assembly sequences that satisfy that logic need to be pursued further, thus making the design process efficient. 


\section{Selection of Assembly Sequence}

Let us say Candidate 3 was chosen for location logic in the previous step. Then there are two possible assembly sequences: $\mathrm{E} \rightarrow \mathrm{A} \rightarrow \mathrm{B} \rightarrow \mathrm{C}$ or $\mathrm{E} \rightarrow \mathrm{C} \rightarrow \mathrm{B} \rightarrow \mathrm{A}$. These two sequences could differ with respect to ease of tolerance achievement. Using the data available about the nominals, tolerances, and process capabilities for the individual parts, one can compute the probability that $Y_{i} \geq 0$ for $i=1,2,3,4$, and decide which sequence is better. For example, if $\mathrm{A}$ has more variability than $\mathrm{C}$, then the second sequence is likely to be better than the first, since the higher variation of A can be transferred to where it is not important. In this case, this is intuitively clear but in complex assemblies, one necessarily needs to carry out such analysis.

\section{Detailed Analysis and Synthesis}

When the design process reaches advanced stages, tolerance analysis and synthesis can be done in a detailed way. For example, given the assembly sequence; detailed specification of nominals and tolerances for A, B, C, and E; and detailed process capability data, one can compute the probabilities associated with each of the four conformance criteria. Also, detailed synthesis or design can be done. This could take one of three forms: optimize nominal dimensions; optimize tolerances; and establish a variance pool that can be distributed across the individual parts.

\subsection{Need and Potential for an Integrated Approach}

The discussion above has brought out the following issues:

1. Continuous evolution of assembly structure and tolerancing information during the design process

2. Close coupling between the design process and tolerancing decisions

3. Availability of a variety of assembly modeling methods at different levels of abstraction and relevant for different stages of the design process

4. Applicability of methods and best practices of design tolerancing to successive stages of the design process.

This motivates the need for and the potential of an integrated approach to design tolerancing that enables tolerancing to be done in a continuous and incremental way.

\section{Assembly Models for Tolerancing}

We first survey relevant assembly models and next look into how some of the assembly models have been used for tolerancing. 


\subsection{Relevant Assembly Models}

There are a variety of assembly models available that capture assembly information at different levels of abstraction during the design process and are useful in specific ways. Assembly representations popularly discussed in the literature and applied in practice are based on solid models, relational models, and hierarchical models [6, 32, 33].

The solid models represent part positions in terms of their spatial coordinates. They provide sufficient information for graphic display of the assembly but are not convenient for purposes of tolerancing. For example, changes to the positions or dimensions of individual parts are not always propagated to neighboring parts in the assembly. According to Mantyla [34] and Whitney [6], geometric models of the type used in most solid models have some limitations:

1. They can represent the product structure at only a single level of abstraction and consequently do not support different kinds of analysis at successive stages of the design process.

2. They lack the capability to record the progression of the design process during various phases and thus cannot capture aspects of design intent.

3. Often, they cannot capture the distinction between essential and non-essential information. For example, they do not distinguish between mates and contacts. Mates are connections that pass dimensional and locational constraints from one part to another. Contacts on the otherhand are all other connections made to provide strength or reinforcement, but not involved in providing locational constraint [5]. Both mates and contacts are important for tolerancing. Mates represent the interfaces to be controlled whereas contacts represent the sources where variation is transferred during assembly.

4. Changes in shape, geometry, and relative positioning to an individual part are not fully propagated to other parts of the model.

5. Geometric data is only one of several attributes of assembly/product data and does not become available until late in the design process. Many fundamental issues in design can be effectively addressed without having to use geometric data.

Relational models represent geometric relations in the form of mating features between individual parts or subassemblies. They are often called liaison diagrams or connective models of assembly [6]. The assemblies are usually modeled as undirected graphs where the nodes represent the parts and the arcs represent the geometric relations between them. The arcs can have annotations such as $\mathbf{P}$ (Part of); A (Attachment); C (Constraint); AS (Assembly), etc. [35]. The actual part or subassembly position can be represented by a coordinate transformation matrix, which is the result 


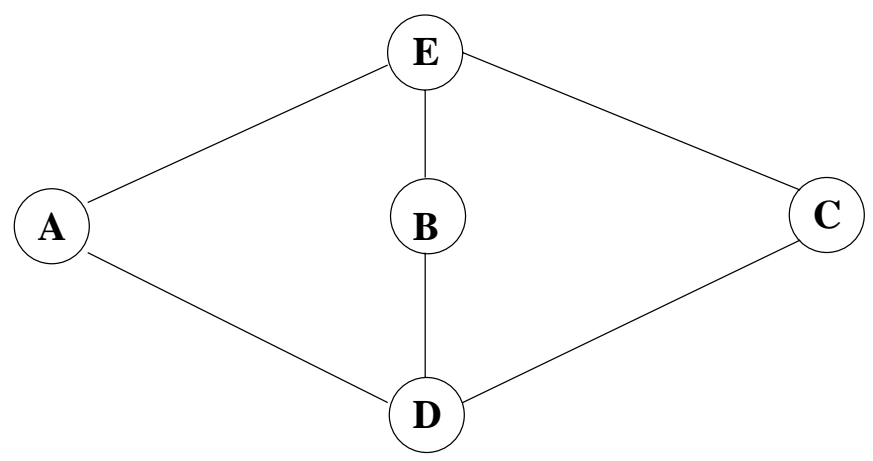

Figure 5: A relational representation of the assembly of Figure 2

of a set of six rigid motions - three translational and three rotational. Figure 5 provides a relational representation of the assembly of Figure 2. It contains five nodes and six arcs in the model. Each arc represents a relation or a liaison between the parts or subassemblies at the two ends of the arc. Relational models cannot capture the order in which the geometrical relationships are established. They have been used in analysis applications such as robot path planning, generation of feasible assembly sequences, and robot assembly planning. [32]. Relational models, by themselves, are not adequate for tolerancing.

In a hierarchical model, an assembly is represented as a collection of subassemblies, which in turn are decomposed into individual parts or next level subassemblies. The actual part or subassembly position can be represented by a coordinate transformation matrix, as in the relational model. Though a hierarchical model captures assembly decomposition and aggregate-level precedence relationships in terms of its different levels, it does not assign any hierarchy on the order of establishment of liaisons between individual parts within a particular subassembly. Also, such a hierarchy is yet undeveloped during early design. A tree structure is most appropriate for representing a hierarchical model. Several variants of the hierarchical model have been employed [32, 36-39]. Figure 6 shows a simple hierarchical representation of the mechanical assembly of Figure 2. The hierarchical model has been used in assembly sequence analysis, kinematics analysis, and tolerance analysis (during advanced stages of design).

A recent proposal for assembly modeling with emphasis on early design representation is that of datum flow chains (DFC) [5,6]. A DFC is a directed acyclic graph that defines the hierarchy of dimensional relationships between parts in an assembly. Each node of a DFC is a part or a fixture or a defined feature on the part or fixture. A directed arc from Node A to Node B indicates that a designated datum corresponding to part A determines the dimensional location of the part B. Dotted lines, if used, (say between nodes $\mathrm{B}$ and $\mathrm{E}$ ) indicate a contact between $\mathrm{B}$ and E. Figure 7 shows a datum flow chain representing a particular way of locating the datums in the mechanical assembly of Figure 2. Assume that each of the five parts, A, B, C, D, and E have 


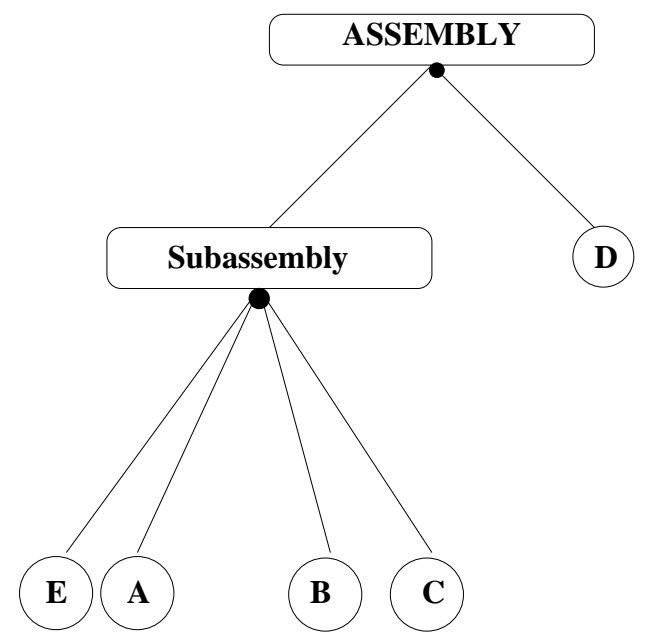

Figure 6: A hierarchical model of the assembly of Figure 2

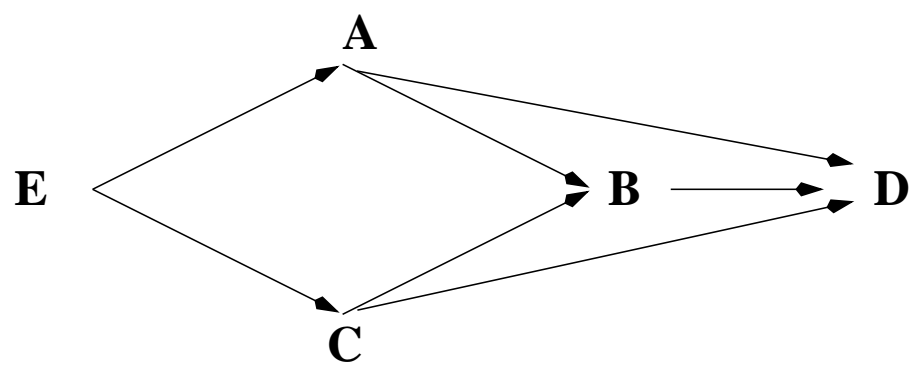

Figure 7: A datum flow chain for the assembly of Figure 2

well-defined datums on them. The location scheme in Figure 7 implies that A and $\mathrm{C}$ are first located with respect to $\mathrm{E}$; $\mathrm{B}$ is then located in reference to $\mathrm{A}$ and $\mathrm{C}$; and the location of $\mathrm{D}$ is decided with reference to the locations of $\mathrm{A}, \mathrm{B}$, and $\mathrm{C}$.

A DFC abstractly captures the underlying location logic of an assembly and often enables a visualization of the way in which tolerance may propagate. DFCs can be used early in the design process to represent evolving assembly configurations. They have been shown to be useful in a variety of ways. For example, they can be used to identify important assembly sequence relationships. Also, when sufficient feature-related information is available, they can be used for deriving tolerance chains of assemblies. If a rough geometrical description (so called skeletal geometry) of the assembly is known, these tolerance chains can be used to conduct tolerance analysis [5].

\subsection{Use of Assembly Models for Tolerancing}

The models discussed above can potentially be used in many ways, such as assembly sequence analysis, kinematics analysis, and tolerance analysis. Since tolerancing is 
the main focus of this work, we now look into the use of these assembly models for tolerancing.

Representation of assemblies for automatic generation of tolerance chains has been described by Wang and Ozsoy [38]. Their model combines relational and hierarchical representations; the assembly is represented in an elaborate data structure with information on assembly decomposition; (4 x 4) transformation matrix for each instance of a component/subassembly; mating features; mating conditions (against, parallel, fit); dimensions and tolerances of the mating features; etc. The above information is used to algorithmically generate a tolerance chain for any given assembly. The chain can be used in tolerance analysis. This representation does not need geometric data but cannot be used in early stages of design due to the nature of information required to complete the data structure.

With tolerance analysis as the main objective, Whitney, Gilbert, and Jastrzebski [40] proposed a model of assembly that contains the following information: Mating features that build up the assembly; a graph representation of mating of parts (liaison diagram); underlying co-ordinate structure of the assembly; and homogeneous (4 x 4) matrix transforms to represent dimensions and tolerances of each part (in accordance with the ASME Y14.5M-1982 geometrical tolerancing standard). The transforms represent both the nominal relations between parts and variations caused by geometric deviations allowed by the tolerances. These transforms can be used to propagate tolerances through an assembly, which allows the location of any designated part to be captured from starting from a reference part, taking into account variations in the locations, sizes, and shapes. The above representation can potentially be used in early stages of design.

Datum flow chains have been used to generate tolerance chains for assemblies during early design [5]. The method uses the location logic embedded in DFCs with skeletal geometry of the assembly, combining it with a $(4 \times 4)$ matrix representation. An important distinction is made between two types of assembly, Type 1 and Type 2, depending on the nature of creation of features [5]. Type 1 assemblies correspond to machined parts, such as automotive engines, and contain parts that arrive at the assembly line with already created assembly features on them. The features have a direct influence on the function of the product. The assembly consists of simply putting the parts together by joining the appropriate features. Type 2 assemblies correspond to items such as car bodies and aircraft structures. Here there may not be any premade assembly features, and non-rigid part geometries are possible. Some of the features are made during assembly with the aid of possibly large and expensive fixtures. The features are decided by assembly needs rather than by functional needs. In Type 1 assemblies, the knowledge of DFC is sufficient to perform a tolerance analysis. This is because all assembly sequences in a family have identical tolerance chains. Hence, if one assembly sequence fails (succeeds) to deliver the tolerance, so will all others corresponding to that family. In Type 2 assemblies, there is scope for in-process 
adjustments. So each assembly sequence within a family can produce different results. This would mean that additional information is required to do tolerance analysis.

There are several other papers that have dealt with the problem of assembly modeling in general and assembly modeling for tolerancing in particular. The reader is referred to $[32,37,41-44]$.

\subsection{Assembly and Tolerance Representation through the De- sign Process}

The following are some important observations about the models for assembly and tolerancing discussed above.

1. Different models become available and are relevant, at possibly different stages of the design process. For example, a relational model becomes available earlier in the design process than a hierarchical model. The models discussed (liaison diagrams, trees, datum flow chains, solid models, etc.), when collectively used, cover a broad spectrum of the design process and therefore are useful for tolerancing at different stages of the design process. See Figure 8 for a preview of the various assembly models.

2. Different models capture the assembly at different levels of abstraction. For example, datum flow chains model design intent related to location logic at a fairly early stage of design. If suitable positioning information is available, DFC models enable tolerance analysis to be done at that (early) stage of design, leading to elimination of difficult or weak designs (difficult from the viewpoint of tolerance achievement).

3. Both the assembly artifact and the tolerancing information evolve during the design process through successive refinement. Consequently, an assembly model continuously evolves through some or all stages of the design process. For example, during early design, not all geometric relations or mating features may be known, so a liaison diagram captures only a subset of all ultimate relations. As the artifact undergoes continuous transformation, existing relations may disappear and new relations can appear, leading to more detailed liaison diagram. Whitney [6] gives an example of how a datum flow chain model evolves as the design function progresses. The key to enabling effective tolerancing to be done at successive stages of the design process lies in a robust assembly model that gets modified and refined in a continuous way throughout the design process.

In our view, an ideal assembly model for tolerancing should have

1. a close coupling with the design process; 
2. should be mutable through successive stages of the design process; and

3. be capable of representing the assembly and tolerance information at any level of abstraction.

Other important attributes of an ideal model would be: capture of design intent; embedding of different views (relational view, location logic view, etc.) in a unifying framework; and enabling all assembly information other than tolerancing, also to be captured in the model. This raises the issue of effective, integrated representations of assembly through the design process. Object oriented models are appealing since they enable such integrated representations of assemblies. There are some recent efforts in this direction. The first is the SHARED model [45-47], which is essentially an information model for cooperative product design. This is an object-oriented representation that captures both an evolving artifact and its associated design process. To represent artifacts as they evolve, the SHARED model defines objects recursively without any pre-defined granularity on the recursive decomposition, thus enabling the model to be used at any desired level of abstraction. The SHARED model, by virtue of a using a single framework to couple the artifact with its design process, provides an attractive paradigm for assembly modeling for continuous tolerancing through the design process. Another effort [48] looks at an object oriented assembly representation that provides a general assembly model that can support both conceptual design at high levels of abstraction and feature modeling at low levels. This is achieved by incorporating functional knowledge and design intent as part of the assembly representation.

Figure 8 summarizes the assembly models for tolerancing. It presents certain selected, representative modeling formalisms only. When supplemented with appropriate information, these models are useful for making tolerance related decisions at different stages of design and constitute an important element of the design for tolerance methodology proposed in this paper.

\section{Design Tolerancing: Methods and Best Practices}

The objective of this section is to provide a brief, global overview of important methods and best practices in tolerancing. These methods and best practices have an important role to play in enabling tolerance-related decisions to be made at successive stages of the design process. As stated earlier, Tolerancing includes both tolerance analysis and tolerance synthesis [49]. In the context of electro-mechanical assembly design, tolerance analysis involves evaluating the effect of variations of individual part or subassembly dimensions on designated dimensions or assembly characteristics of the resulting assembly. Tolerance synthesis involves allocation of tolerances to individual parts or subassemblies of an assembly based on the tolerance requirements on the assembly. 


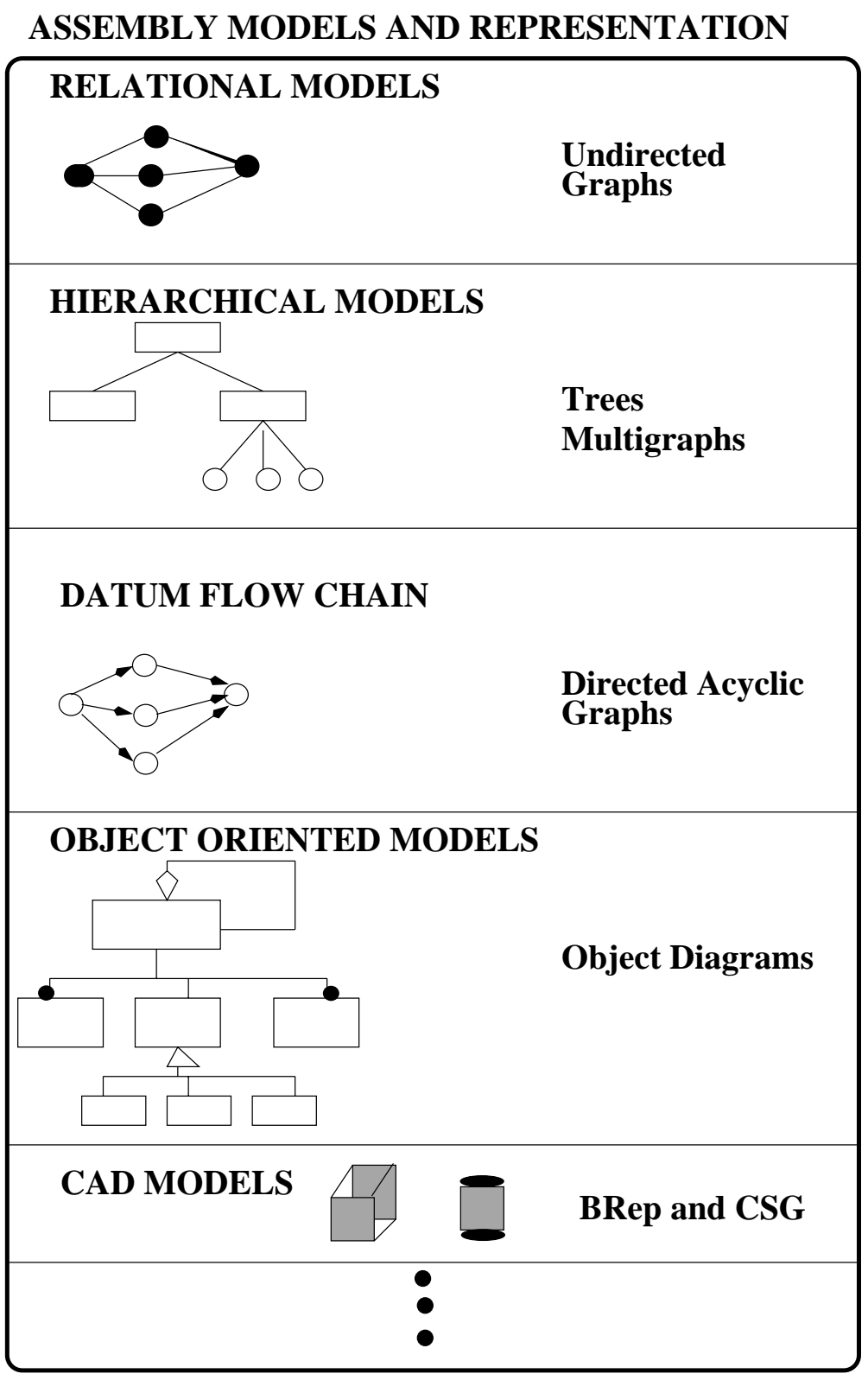

Figure 8: Assembly models for tolerancing 
We first present important methods for tolerance analysis. These include: worstcase tolerancing; linearization (or root sum of squares); extended Taylor series; numerical integration based on quadrature techniques; and Monte Carlo simulation. The material for these methods is culled out from the articles by Evans [13], Chase and Parkinson [15], and Nigam and Turner [18]. Next, we look into tolerance synthesis methods. A common approach to tolerance synthesis is to use tolerance analysis in an iterative way. Thus, all tolerance analysis approaches become relevant for tolerance synthesis. Other methods on synthesis include: mathematical programming and heuristic optimization techniques; and design of experiments. The principal sources for this topic are the articles by Evans [13] and by Kusiak and Feng [19]. We then briefly review three industry best practices in tolerancing: The Motorola Six Sigma Program [20, 50, 51]; the Xerox Holistic Probabilistic Design Methodology [52, 53], and the Taguchi robust design methodology [21,54,55]. Figure 9 shows a listing of important methods for tolerance analysis and synthesis, and major best practices.

\subsection{Methods for Tolerance Analysis}

Tolerance analysis can be either worst-case or statistical. In worst-case tolerance analysis (also called deterministic or high-low tolerance analysis), the analysis considers the worst possible combinations of individual tolerances and examines the assemblability of the parts, so as to achieve $100 \%$ interchangeability of parts in an assembly. This results in unnecessarily tight part tolerances and hence high production costs. Statistical tolerancing is a more practical and economical way of looking at tolerances and works on setting the tolerances so as to assure a desired yield. Here, the designer abandons the notion of $100 \%$ interchangeability and accepts some small percent of non-conformance.

Statistical tolerance analysis uses a relationship of the form:

$$
Y=f\left(X_{1}, \ldots, X_{n}\right)
$$

where $Y$ is the response (a measurable characteristic such as assembly gap) of the assembly and $X_{1}, \ldots, X_{n}$ are the values of some characteristics (such as dimensions) of the individual parts or subassemblies making up the assembly. We call $f$ the assembly response function $(\mathrm{ARF})$. The relationship can exist in any form for which it is possible to compute a value for $Y$ given values of $X_{1}, \ldots, X_{n}$. It could be an explicit analytic expression or an implicit analytic expression, or could involve complex engineering calculations or conducting experiments or running simulations. The input variables $X_{1}, \ldots, X_{n}$ are continuous random variables. In general, they could be mutually dependent. The function $f$ is a deterministic relationship; $Y$ is easily seen to be a continuous random variable. The general problem of tolerance analysis is to compute the probability distribution of $Y$ given the distributions of $X_{1}, \ldots, X_{n}$. However, more often we are usually interested in computing the first few moments ( or mean, standard deviation, skewness, and kurtosis), given the distributions or first few moments of the 
Methods and Best Practices for Tolerancing

\begin{tabular}{|c|c|}
\hline & METHODS \\
\hline \multirow{3}{*}{$\begin{array}{l}\text { Methods } \\
\text { for } \\
\text { Analysis }\end{array}$} & Worst Case \\
\hline & Linearization (Root Sum of Squares - RSS) \\
\hline & Extended Taylor Series Approximation \\
\hline \multirow{6}{*}{$\begin{array}{l}\text { Methods } \\
\text { for } \\
\text { Synthesis }\end{array}$} & Numerical Integration (Quadrature Techniques) \\
\hline & Monte Carlo Simulation \\
\hline & Based on Analysis Methods (applied iteratively) \\
\hline & Mathematical Programming \\
\hline & Heuristic Optimization \\
\hline & Design of Experiments \\
\hline \multicolumn{2}{|r|}{ BEST PRACTICES } \\
\hline \multicolumn{2}{|c|}{ Motorola Six Sigma } \\
\hline \multicolumn{2}{|c|}{ Taguchi Methods $^{(\mathrm{TM})}$} \\
\hline \multicolumn{2}{|c|}{ Xerox Holistic Probabilistic Design } \\
\hline & ific Industry Best Practices \\
\hline
\end{tabular}

Figure 9: Methods and best practices for tolerancing 
input variables. Once the moments of $Y$ are determined, one can compute a tolerance range for $Y$ that would envelope a given fraction of the assembly yield.

There are a variety of methods and techniques available for the above computational problem. Essentially, the methods can be categorized into four classes [13]:

1. Stack Tolerancing or Linear Propagation (Root Sum of Squares)

2. Non-linear propagation (Extended Taylor series)

3. Numerical integration (Quadrature technique)

4. Monte Carlo simulation

\subsubsection{Linear Propagation}

This is also called as stack tolerancing and uses the well-known root sum of squares (RSS) formula. The assembly response function here is of the form:

$$
Y=a_{0}+a_{1} X_{1}+a_{2} X_{2}+\ldots+a_{n} X_{n}
$$

where $a_{0}, a_{1}, \ldots, a_{n}$ are constants and $X_{1}, \ldots, X_{n}$ are assumed to be mutually independent. Many dimensional and gap-related measures fall into this category. Because of the linear relationship and mutual independence, the mean and variance of $Y$ are given by:

$$
\begin{aligned}
\mu_{Y} & =a_{0}+a_{1} \mu_{1}+a_{2} \mu_{2}+\ldots+a_{n} \mu_{n} \\
\sigma_{Y}^{2} & =a_{1}^{2} \sigma_{1}^{2}+a_{2}^{2} \sigma_{2}^{2}+\ldots+a_{n}^{2} \sigma_{n}^{2}
\end{aligned}
$$

where $\mu_{i}$ is the mean and $\sigma_{i}$, the standard deviation of $X_{i}, i=1, \ldots, n$. The nomenclature RSS arises because of the formula above for standard deviation. If the individual distributions are normal, then $Y$ is also normally distributed. Even if the individual distributions are not normal, $Y$ can safely be treated as normal, by invoking the central limit theorem.

If the linear relation for $Y$ above is only approximately true, then one can expand $f\left(X_{1}, \ldots, X_{n}\right)$ as a Taylor series and drop all but the constant and linear terms. This is often-used device in statistical tolerancing to handle approximately linear relationships. In such a case,

$$
a_{i}=\frac{\partial f}{\partial x_{i}} \text { evaluated at } x_{i}=\mu_{i}, i=1, \ldots, n,
$$

and all of the constant terms are gathered into $a_{0}$. The computation of the above partial derivatives could be of two types. In the first case, the function $f$ is known and the partial derivatives are known to exist. In the second case, the functional relationship is either too intractable or not even available in analytic form. In such a case, numerical estimates have to be obtained for the partial derivatives [13]. 
The linear case is easily the simplest and the most efficient among all tolerance analysis approaches. It is very appealing for synthesis methods that use analysis in an iterative way.

\subsubsection{Non-linear Propagation (Extended Taylor Series)}

If the assembly response function $Y$ is highly non-linear, application of the RSS method could lead to serious errors. In such a case, an extended Taylor series approximation for the relationship $f$ can possibly be employed. For this, $f$ needs to be available in analytic form. Usually, the expansion is considered up to sixth order. The expansion is possible only when all the appropriate partial derivatives exist. The main computational issue here is that of computing the partial derivatives. Tractable formulae for the first four moments of $Y$ are available [13] and are ideally suited for tolerance analysis and synthesis. These formulae need only the first four moments of the distributions of $X_{1}, \ldots, X_{n}$. Most often, the partial derivatives are computed using analytic methods. However, numerical evaluation may need to contemplated in some cases (in such cases, the quadrature technique, discussed next, is more appropriate).

\subsubsection{Numerical Integration}

If the function $f$ is not available in analytic form and $Y$ can only be computed through numerical calculations or engineering methods or simulations, numerical methods have to be used. Quadrature methods are prominently used here. The basis of the numerical methods is that for any function $h\left(X_{1}, \ldots, X_{n}\right)$ (different from $f$ ) of mutually independent random variables $X_{1}, \ldots, X_{n}$ with probability density functions $w_{X_{i}}\left(x_{i}\right)$, the expected value of $h$ is given by the integral

$$
\int_{-\infty}^{+\infty} \ldots \int h\left(x_{1}, \ldots, x_{n}\right) \prod_{i=1}^{n}\left(w_{X_{i}}\left(x_{i}\right) d x_{i}\right)
$$

The above expression can be approximated by a quadrature expression [13] that involves evaluations of $h$ at $2 n^{2}+1$ prescribed values. These evaluations involve only the first four moments of $X_{1}, \ldots, X_{n}$. Given an assembly response function $f$, a corresponding function $h$ as above can be defined and simple moment transfer relations can be used to compute the first four moments of $f$. The quadrature technique adapts well to statistical tolerancing problems since it can handle the iteration inherent in a tolerancing problem efficiently. The HPD [52,53] can handle this integration in a much better way.

\subsubsection{Monte Carlo Simulation}

The appeal of Monte Carlo lies in its applicability under very general settings and the unlimited precision that can be achieved. In particular, Monte Carlo can be used in 
all situations in which the above three techniques (stack tolerancing, extended Taylor series, and numerical integration) can be used and can yield more precise estimates. For this reason, Monte Carlo technique is easily the most popular tool used in tolerancing problems. The caveat, however, is the large computational time. For situations where the above three techniques are adequate and have acceptable precision, the Monte Carlo technique is much more expensive in terms of computational time.

Monte Carlo analysis proceeds as follows. Pseudo random number generators are used to generate a sample of numbers $x_{1}, \ldots, x_{n}$, belonging to the random variables

$X_{1}, \ldots, X_{n}$, respectively. The value of $Y$, say $y_{1}=f\left(x_{1}, \ldots, x_{n}\right)$, corresponding to this sample is computed. This procedure is replicated a large number of times, say $N$ times. This would yield a random sample, $\left\{y_{1}, \ldots, y_{N}\right\}$ for $Y$. Standard statistical estimation methods are then used to analyze the distribution of $Y$. The precision of this statistical analysis increases proportional to $\sqrt{N}$ and therefore unlimited precision can be achieved through large number of replications. Special techniques are available for significantly enhancing the precision of the Monte Carlo method for a given $N$. These include: weighted sampling, reuse of samples, and use of approximation functions [13].

\subsection{Methods for Tolerance Synthesis}

In the context of electro-mechanical assembly design, tolerance synthesis usually refers to the allocation of specified assembly tolerances among the constituent parts and subassemblies, so as to ensure a specified yield or minimize a proper cost function. More generally, if $Y=f\left(X_{1}, \ldots, X_{n}\right)$ is an assembly response function, then the synthesis problem involves finding the best nominals and tolerances for $X_{1}, \ldots, X_{n}$, given nominal and tolerance specifications for $Y$. Synthesis is naturally an optimization problem; one can formulate an objective function that captures yield requirements or production cost requirements and pose an optimization problem by including tolerance related constraints.

There are several views and variants of the synthesis problem, depending on the objective function and the constraints. One view is to minimize the total manufacturing cost by choosing both the individual part tolerances and the manufacturing processes for making the individual parts. This requires cost versus tolerance relationships for each individual dimension. Another view is to find robust nominals for individual dimensions, i.e. nominal values at which the effect of variations on the assembly response function is minimum. This is the problem addressed by Taguchi's robust design methodology and HPD. Also, depending on the nature of the objective function and the constraints, the synthesis problem can be deterministic or stochastic.

To formulate the synthesis problem meaningfully, a certain amount of preprocessing is often required. For example, one needs to first determine the tolerance limits on the assembly response function, $Y$. An important preprocessing step is sensitivity analysis, that determines which assembly parameter variations have significant effects 
on the assembly response function. This reveals the set of parameters or individual dimensions to emphasize in the synthesis procedure.

\subsubsection{Iterative Methods based on Analysis}

A simple and realistic mechanism for tolerance synthesis lies in employing a trial and error technique for postulating tolerances for individual parts and subassemblies and then performing a statistical tolerance analysis required to ascertain whether this postulated set of tolerances fulfills the desired criteria. If the chosen set is unsatisfactory, some of the tolerances are changed and the analysis redone; this step is repeated until a satisfactory set of part tolerances are obtained. Typically, at the end of each iteration, we obtain a probability of assembly, probability of conformance, expected yield, or a more detailed cost. The appeal of this technique lies in the scope for effecting trade-offs during each iteration and in the scope for using the findings of the current iteration to drive the next iteration. During early iterations, approximate cost figures and less accurate estimates can be used and these can be replaced by more accurate figures as the iterations start producing good solutions.

The methods discussed for statistical tolerance analysis, namely stack tolerancing, extended Taylor series, quadrature methods, and Monte Carlo, are all suited for the iterative approach. Evans [13] has discussed the merits and issues concerning the use of these methods from the angle of an iterative methodology for synthesis.

\subsubsection{Optimization Methods}

Since tolerance synthesis can be posed as an optimization problem, mathematical programming techniques such as linear programming, non-linear programming, and, integer programming are relevant. There have been several efforts in this direction $[15,19,56]$. Also, heuristic techniques for optimization such as simulated annealing, genetic algorithms, Lagrangian relaxation, and Tabu search have been used by researchers [57-59].

To give a flavor of a typical synthesis problem in the optimization framework, we show below the integer programming formulation given by Kusiak and Feng [19]:

Minimize

$$
\sum_{i=1}^{n} \sum_{j=1}^{m} c_{i j} x_{i j}
$$

subject to:

$$
\begin{aligned}
& \sum_{i=1}^{n} \sum_{j=1}^{m} t_{i j} x_{i j} \leq T \\
& \sum_{j=1}^{n} x_{i j}=1 \quad \forall i
\end{aligned}
$$




$$
x_{i j}=0,1 \quad \forall i, j
$$

where the index $i$ denotes one of $n$ dimensions involved in the assembly; index $j$ denotes one of $m$ manufacturing processes that can be used for producing a dimension; $c_{i j}$ is the manufacturing cost of process $j$ when used for producing dimension $i$; $t_{i j}$ is the $3 \sigma$ normal variation of process $j$ when used to produce dimension $i$; $T$ is the tolerance stackup limit for the assembly; and $x_{i j}$ is a binary decision variable that takes a value 1 if process $j$ is selected for producing dimension $i$ and 0 otherwise. Note that the objective is to minimize the total direct manufacturing cost, by choosing the appropriate tolerances and the right mix of manufacturing processes. In the above formulation, a linear relationship has been assumed between part tolerances and also worst case tolerancing has been used. Thus the above is a deterministic tolerance synthesis problem. In HPD, the optimization is done considering the nominals and variance together.

\subsubsection{Design of Experiments}

Here, the assembly response function (or in general, a well-defined cost function), is computed for various discretized values of the random variables $X_{1}, \ldots, X_{n}$, (dimensions with tolerances) according to the theory of design of experiments. The factors used in the experiment include not only the individual values of $X_{1}, \ldots, X_{n}$, but also factors that capture tolerance related constraints. Full factorial or fractional factorial designs can be used depending on the number of factors and levels of the factors. Prior sensitivity analysis can throw light on which and how many levels to use for the factors. The setting that leads to the minimum cost and also satisfies the tolerance constraints can be chosen as the solution. One can go a step further and estimate a statistical model that describes the cost function in terms of all the factors and use this model to arrive at an optimal solution for the problem.

Taguchi methods, which are described in the next section, use design of experiments in a novel way to find robust nominals.

\subsection{Best Practices}

In the last decade, many companies have established comprehensive programs in total quality management. These efforts include those of Motorola, Xerox, IBM, AT \& T Bell Laboratories, and several others which have initiated formal, corporate programs for improved tolerance specification, monitoring, and control. In this section, we outline the tolerancing best practices at Motorola and Xerox. We also provide an overview of Taguchi's robust design methodology, which has emerged as a best practice in many companies. 


\subsubsection{Motorola Six Sigma Program}

Six sigma quality is the benchmark of excellence for product and process quality, popularized by Motorola [20,50]. It provides a quantitative, statistical notion of quality useful in understanding, measuring, and reducing variation. A product is said to be of six sigma quality if there are no more than 3.4 non-conformities per million opportunities $(3.4 \mathrm{ppm})$ at the part and process-step level, in the presence of typical sources of variation. The six sigma quality concept recognizes that variations are inevitable due to insufficient design margin, inadequate process control, imperfect parts, imperfect materials, fluctuations in environmental conditions, operator variations, etc.

Tolerance analysis and synthesis in the six sigma program are based on:

1. six sigma characterization of products and processes; the process capability indices $C_{p}$ and $C_{p k}$ are used as the vehicles to characterize the product-process quality;

2. simple, intuitive extensions to the RSS method, to enable tolerance analysis and synthesis in the presence of shifts and drifts of the process mean; and

3. a well-defined, systematic program for design for quality, taking into account both the product perspective and the process perspective.

We provide a brief outline of the first two issues above; a detailed discussion of these and the third issue can be found in [20,50,51].

\section{Process Capability Indices}

Let $U$ and $L$ be the upper and lower specification limits, respectively, of a part dimension or a product attribute. Assume that $\sigma$ is the standard deviation of the process that produces the dimension. Then, the index $C_{p}$ is defined as:

$$
C_{p}=\frac{U-L}{6 \sigma}
$$

The numerator above represents the specification width whereas the denominator captures the total width of the $3 \sigma$ limits of the process distribution. For the rest of the discussion, assume that the process is normally distributed. The denominator then represents $99.73 \%$ limits for the process distribution. If $C_{p}=1$, the implication is that the specification width is the same as the distribution width and when the process mean is centered at $\left(\frac{U+L}{2}\right)$ without any shift, the probability that the actual dimension is within the specification limits is 0.9973 (2700 ppm defect rate). Similarly, if $C_{p}=2$, we have that the specification width is twice that of the distribution. In this case, when the process mean is centered at $\left(\frac{U+L}{2}\right)$ without any shift, the probability 
of conformance is 0.999999998 (.002 ppm defect rate). Since $\left(\frac{U-L}{2}\right)$ is the tolerance $T$ of the part dimension (or in general of any attribute of a product), we have that:

$$
\sigma=\frac{U-L}{6 C_{p}}=\frac{T}{3 C_{p}}
$$

The index $C_{p}$ does not capture how far away the process mean $\mu$ is from the ideal value $\tau$ (target value). The Motorola six sigma program assumes that the ideal value of the process mean is the midpoint of the specification interval, i.e. $\left(\frac{U+L}{2}\right)$. The index $C_{p k}$ captures the effect of the shift in the process mean in the following way:

$$
C_{p k}=C_{p}(1-k) \quad \text { where } k=\frac{|\tau-\mu|}{\left(\frac{U-L}{2}\right)}
$$

The factor $k$ above can be interpreted as the fraction of tolerance consumed by the mean shift. The above definition of $C_{p k}$ assumes that $\tau=\frac{U+L}{2}$ and for a general definition, refer [60].

The Motorola convention is to use a one sided mean shift of $1.5 \sigma$. The one sided mean shift is perhaps motivated by common physical phenomena such as tool wear. A shift of $1.5 \sigma$ is motivated by earlier work by Bender [61]. Also, it is assumed that the process standard deviation is invariant.

If $C_{p}=2$ and $C_{p k}=1.5$ (mean shift consumes 25 percent of the tolerance range), the probability of conformance can be shown to be 0.9999966 , which is equivalent to $3.4 \mathrm{ppm}$. Thus $C_{p} \geq 2$ and $C_{p k} \geq 1.5$ imply six sigma quality, assuming a $1.5 \sigma$ one sided mean shift.

\section{Tolerance Analysis and Synthesis in the Motorola Approach}

The Motorola program assumes a linear model for $Y$, of the form

$$
Y=a_{0}+a_{1} X_{1}+a_{2} X_{2}+\ldots+a_{n} X_{n}
$$

If there is no mean shift, then the standard RSS formulae are applicable:

$$
\begin{aligned}
\mu_{Y} & =a_{0}+a_{1} \mu_{1}+a_{2} \mu_{2}+\ldots+a_{n} \mu_{n} \\
\sigma_{Y}^{2} & =a_{1}^{2} \sigma_{1}^{2}+a_{2}^{2} \sigma_{2}^{2}+\ldots+a_{n}^{2} \sigma_{n}^{2}
\end{aligned}
$$

Recall that $\sigma_{i}$, for $i=1, \ldots, n$, can also be written as:

$$
\sigma_{i}=\frac{T_{i}}{3 C_{p i}}
$$

where $T_{i}$ is the tolerance range of the $i$ th part and $C_{p i}$ is the $C_{p}$ value for the $i$ th part $(i=1, \ldots, n)$. In the presence of a mean shift, the standard RSS formula cannot 
be used for computing the standard deviation of $Y$. Two alternative approaches are recommended by the Motorola program. The first is the Dynamic RSS where the $C_{p k}$ values, $C_{p k 1}, \ldots, C_{p k n}$, of the individual processes corresponding to dimensions $X_{1}, \ldots, X_{n}$; and the tolerances, $T_{1}, \ldots, T_{n}$, of the individual parts, are used in the following way to compute the variance of $Y$ :

$$
\sigma_{Y}^{2}=a_{1}^{2}\left(\frac{T_{1}}{3 C_{p k 1}}\right)^{2}+\ldots+a_{n}^{2}\left(\frac{T_{n}}{3 C_{p k n}}\right)^{2}
$$

Note that the standard deviations $\sigma_{i}$ are inflated by an amount equal to $\frac{C_{p i}}{C_{p k i}}$, for $i=1, \ldots, n$. Thus the dynamic RSS method emulates random behavior in the process mean by inflating the process standard deviation. The second alternative method, called Static RSS, does this emulation by applying a correction factor to the individual nominals. For details, see [50].

Tolerance analysis is carried out by using RSS, dynamic RSS, and static RSS, as appropriate. Tolerance synthesis employs the common approach of using tolerance analysis in an iterative way. Each iteration will evaluate the resulting probability of non-conformance and the $C_{p}$ and $C_{p k}$ values. The synthesis procedure seeks to obtain a probability of non-conformance of at most $3.4 \mathrm{ppm}$, which is guaranteed by $C_{p} \geq 2$ and $C_{p k} \geq 1.5$. The synthesis can assume several forms:

1. Finding optimal values for nominal dimensions

2. Finding optimal values for tolerances

3. Establishing a variance pool that can be allocated to individual processes so as to obtain the desired assembly yield

The Motorola six sigma approach critically uses the normal distribution for all its probability and tolerancing computations. While this can be listed as a limitation, it takes very little away from the intrinsic novelty and applicability of the approach. The ideas it has germinated essentially hold in all situations; only the probability computations need to be redone under non-normal situations and the quantitative measures need appropriate reinterpretation.

\subsubsection{Xerox Holistic Probabilistic Design}

The HPD methodology ${ }^{1}$ is one of several quality programs at the Xerox Corporation. The program is based on relating service dissatisfiers and customer tolerances to a set of critical parameters (parameters that are critical to the product's function). The tolerances of the critical parameters are related to piece part variabilities through

\footnotetext{
${ }^{1}$ HPD is owned by J.M.Parks and University of Rochester
} 
multiple flow of variance chains. Tolerance analysis and synthesis are carried out through the chains to yield the desired quality. Since the objective is to maximize the amount of manufacturing and usage variability the product can tolerate, with negligible impact on the targeted level of performance, the program is also called design for latitude. The methodology is implemented using a complete suite of tools for stochastic variability analysis. These include:

1. A stochastic modeling based technique for computing the distribution of a function of a random variables almost exactly

2. Contribution analysis that provides a reliable guidance of which factors have significant effect on the assembly response function

3. An operating window optimization method that helps choose the densities of certain input random variables for which the allowable range of operation is maximized

Tolerance analysis is based on a stochastic technique that uses a failure rate prediction model. Let $Y=f\left(X_{1}, \ldots, X_{n}\right)$ be the assembly response function, as usual. If failure is defined by $f\left(X_{1}, \ldots, X_{n}\right)>Y_{0}$, then the probability of failure is given by:

$$
\operatorname{Pr}\left\{f\left(X_{1}, \ldots, X_{n}\right)>Y_{0}\right\}=\int \ldots \int_{\Omega} w\left(X_{1}, \ldots, X_{n}\right) d X_{1} \ldots d X_{n}
$$

where $\Omega$ is the $n$-dimensional failure region and $w\left(X_{1}, \ldots, X_{n}\right)$ is the joint density of the $n$ random variables. From this it is easy to see how to compute the distribution function of $Y$. Assuming mutual independence of $X_{1}, \ldots, X_{n}$, the HPD tool uses an efficient numerical technique to evaluate such multiple integrals. This enables us to compute the distribution almost exactly. This computation is versatile since it can handle any type of distribution and any type of relationship; it has excellent computational performance if the number of random variables is less than 10. The above computation enables variability analysis and hence tolerance analysis and also provides a sound basis for iterative tolerance synthesis. An attractive feature of this technique is its applicability to both geometric and non-geometric type of situations. For example, the random variables $X_{1}, \ldots, X_{n}$ need not be dimensions; they could be physical quantities such as force, pressure, temperature, and speed.

The contribution analysis embedded within HPD is a powerful feature of HPD. It provides a sound basis for determining the input variables that have a pronounced effect on the assembly response function. Also, it takes into account the nature of the input distributions and accounts for cross-term effects. This makes it superior to existing techniques for contribution analysis.

Another feature of HPD is the stochastic operating window optimization. This feature enables us to maximize the allowable range of operation by intelligently selecting the densities of input random variables using the tools provided by HPD. 
The HPD tool comprises two major modules: HPD-VA and HPD-OPT. The module HPD-VA is a stochastic analyzer that includes variability analysis and tolerance analysis. HPD-OPT has a wide variety of deterministic, stochastic, and statistical optimization routines. HPD-OPT finds the most robust set of nominals and tolerances.

\subsubsection{Taguchi Methods}

Taguchi methods, also known as robust design methods, [21, 54, 55], are technical methods for quality and cost control at the product and process design stages. According to Taguchi, the cost of a product is the loss incurred to the society before the product is shipped to the customer, whereas quality is the loss imparted to the society after the product has been shipped to the customer. Such losses include: loss due to harmful side effects; loss due to variations in the product's performance characteristics; and all losses that can be traced to the poor performance of the product. Taguchi methods emphasize reducing the sensitivity of engineering designs to various sources of variation. The methods are cost-effective since the idea is to minimize influence of sources of variation rather than control them.

Let $Y$ be a performance characteristic; as before, $Y$ is a continuous random variable. Taguchi considers $Y$ as a function of design parameters or design factors, $\Theta=\left(\theta_{1}, \ldots, \theta_{k}\right)$, and noise parameters or noise factors, $W=\left(w_{1}, \ldots, w_{t}\right)$. Thus, $Y=f(\Theta, W)$. Design factors are input variables whose nominal settings can be chosen by the designer and that have pronounced influence on $Y$. Design factors are of two types: Signal factors, which affect only the mean of $Y$, and control factors, which affect both mean and variance of $Y$. Noise factors are input variables that cause $Y$ to deviate from its target value. Noise factors include deviations of the actual values of design factors from the nominal settings. Taguchi considers two types of matrices, the design matrix (inner array) and the noise matrix (outer array). The design matrix has $k$ columns, each column corresponding to a particular design factor. Each row of this matrix represents a specific combination of design parameter settings. The number of rows depends on the number of combinations of design parameter settings sought to be investigated. Similarly, the noise matrix has $t$ columns, each column corresponding to a particular noise factor. Each row of this matrix represents a specific combination of noise factor settings. The number of rows depends on the number of combinations of noise factor settings sought to be investigated.

Let $\tau$ be the target value (ideal value) of $Y$; $\mu$, its mean; and $\sigma$, its standard deviation. The target value need not be the midpoint of a tolerance interval. Variations of $Y$ about the target value $\tau$ cause losses to the customers. Let $l(Y)$ be the loss due to deviation of $Y$ from $\tau$. Taguchi suggests a quadratic form for the loss function:

$$
l(Y)=k(Y-\tau)^{2}
$$

where $k$ is a constant that can be computed from a known value of loss at any designated value of $Y$. The loss function is a random variable and the expected quadratic loss, 
$E[l(Y)]$, is given by

$$
L=E[l(Y)]=k\left(\sigma^{2}+(\mu-\tau)^{2}\right)
$$

Thus the expected quadratic loss is the the sum of the variance of $Y$ and the square of bias (bias indicates how far away from the target value the process mean is behaving).

Minimization of the expected quadratic loss is the primary objective of Taguchi methods. This is accomplished by maximizing a signal-to-noise ratio (also called as a performance statistic). Taguchi's use of signal-to-noise ratios rather than directly the expected quadratic loss is motivated by considerations such as ease of statistical estimation, more direct coupling to design factors, and improved additivity of control factor effects. See $[21,55]$ for a detailed exposition. A signal-to-noise ratio is a statistical estimate of the effect of noise factors on $Y$ for a particular setting of design parameters. Numerous performance statistics have been defined by Taguchi (more than 60).

The following are the main steps in the Taguchi method.

1. Identify appropriate loss function or signal-to-noise ratio, initial and competing settings of design factors, and, important noise factors and their ranges.

2. Construct the design matrix and the noise matrix. The design matrix is chosen based on the theory of design of experiments or is chosen from Taguchi's collection of orthogonal arrays [21]. The noise matrix is usually chosen from Taguchi's collection orthogonal arrays.

3. Conduct a parameter design experiment. This involves $N_{d}$ runs, where $N_{d}$ is the number of rows of the design matrix. Each run corresponds to a particular row and involves $N_{n}$ replications, where $N_{n}$ is the number of rows of the noise matrix. For each run, $i,\left(i=1, \ldots, N_{d}\right)$, a corresponding signal-to-noise ratio, $[Z(\Theta)]_{i}$ is computed.

4. Use $[Z(\Theta)]_{1}, \ldots,[Z(\Theta)]_{N_{d}}$, to predict a statistical model for the signal to noise ratio. Use the model to obtain optimal or best design parameter settings:

$$
\Theta^{*}=\left(\theta_{1}^{*}, \ldots, \theta_{k}^{*}\right)
$$

5. Conduct a verification experiment to confirm that $\Theta^{*}$ indeed minimizes the expected loss. Otherwise, iterate.

Taguchi methods are based on assumptions such as: absence of interaction effects among the factors; additivity of control factors; separability of signal factors and control factors; and use of signal-to-noise ratios instead of direct measures [21, 55]. However, the methodology embodies sound engineering considerations and intuition for obtaining robust designs; which explains its widespread use. From a tolerancing viewpoint, Taguchi methods provide a valuable tool for synthesizing robust nominals. Also, the 
methods can be applied potentially during early stages of assembly design. Furthermore, the methods enable economic considerations to be incorporated into tolerance analysis and synthesis, and provide an approach that is complementary in many ways to other best practices such as the Motorola six sigma program and the Xerox HPD methodology.

\section{Design for Tolerance Process}

We now propose an integrated approach, which we call Design for Tolerance, for enabling tolerancing decisions in an incremental and continuous ongoing fashion during the design of assemblies.

\subsection{Design Process for Assemblies}

In the literature, several researchers have presented their viewpoint of what the assembly design process should be. We provide a brief outline of some viewpoints that emphasize tolerancing. Whitney [6] advocates top-down design of assemblies, which uses the method of key characteristics (KCs) [3-5]. Customer requirements or customer tolerances are specified in terms of product key characteristics (PKCs); which are permanent properties of the design. These flow down to the subassembly and part levels resulting in a set of assembly key characteristics (AKCs) and a set of manufacturing key characteristics (MKCs). AKCs define important dimensional datums, assembly mating features, and fixturing features on parts and assemblies [6]. These are defined in the context of a specific assembly process that is intended to deliver the PKCs. MKCs are basically parameters of manufacturing processes that are intended to deliver the AKCs. Design of the assembly is driven by the KCs and implemented using datum flow chains. Tolerance analysis forms an important part of verifying whether or not the key characteristics are delivered by the chosen location logic or assembly sequences.

Tolerancing best practices discussed in Section 4.3 also advocate their own design processes for assemblies. The Motorola six sigma program [50] has a five-step process:

1. Perform preliminary design: Starting from customer specifications, establish a baseline design and develop a basic configuration. This will involve choosing baseline nominals for important dimensions.

2. Identify process variabilities.

3. Assign tolerances to related dimensions.

4. Compute the probability of conformance for each assembly gap and assembly response measure. 
5. Optimize the probability of conformance for each assembly gap and assembly response measure. This may involve finding optimal nominals, determining best tolerances, and distributing the overall assembly variation among individual parts of the assembly. Confirm six sigma quality with respect to all the assembly gaps and assembly response measures.

The Xerox HPD methodology recommends the use of critical parameters that are derived from customer specifications and customer tolerances [53]. The critical parameters are similar to key characteristics. The critical parameters are systematically related to piece-part variabilities through flow-of-variance chains. Tolerance analysis and synthesis involve choosing the piece part variabilities so as to yield the customer desired tolerances for all the assembly response measures.

Taguchi's robust design process follows a three-step approach [21]: system design, parameter design, and tolerance design. In system design, a basic functional prototype is designed after understanding the customer's needs and the manufacturing environment. In parameter design, settings of product or process parameters that minimize the sensitivity of designs to the sources of variation are obtained. These settings are called robust nominals. In tolerance design, tolerances around the robust nominal settings are determined.

The SIMA (Systems Integration for Manufacturing Applications) reference architecture formulated at the National Institute of Standards and Technology [62] provides a generic specification of design related activities for electro-mechanical products. Figure 10 shows the various design stages and activities in the SIMA reference architecture. Stage A11 (Plan Products) involves developing the idea for the assembly depending on market needs and customer requirements and characterizing it in terms of function, target price range, and relationship to existing product lines. In Stage A12 (Generate Product Specifications), an engineering specification for the assembly is formulated. This involves mapping the customer requirements into engineering requirements and refining these in consideration of the relevant laws, regulations, patents, and product standards, etc. In Stage A13 (Perform Preliminary Design), the assembly design problem is decomposed into a set of component/subassembly design problems and specifications are developed for each component/subassembly problem. Interface specifications between the components/subassemblies are developed and a preliminary assembly layout is created. Finally, in Stage A14 (Produce Detailed Designs), all specifications needed to completely describe each subassembly or component are produced. This includes drawings and geometry, materials, finish requirements, assembly drawings, and fit and tolerance requirements.

There are several commonalities in the SIMA reference architecture and the assembly design processes outlined earlier. The design for tolerance process proposed in this paper embodies many of these ideas in the broad framework of the SIMA architecture, with emphasis on tolerancing. 


\title{
A1 : DESIGN PRODUCT
}

\section{A11 : Plan Products}

\section{A12 : Generate Product Specifications}

\section{A13 : Perform Preliminary Design}

A131 : Develop Functional Decompositions

A132 : Evaluate and Select Decompositions

A133 : Develop Preliminary Configurations

A134 : Consolidate Configurations

A135 : Evaluate Alternative Designs

A136 : Select Design

\section{A14 : Produce Detailed Designs}

\author{
A141 : Design System/Component \\ A142 : Analyze System/Component \\ A143 : Evaluate System/Component Design \\ A144 : Optimize Designs \\ A145 : Produce Assembly Drawings \\ A146 : Finalize System/Component Design
}

Figure 10: Design stages and activities in the SIMA reference architecture. Source: NIST Internal Report 5939 [62]

\subsection{Design Tolerancing: An Incremental Process}

Potentially, tolerance considerations can influence the decisions taken at different design stages, in increasing level of detail. Also, the decisions taken at a particular stage influence and can simplify the decisions taken in the downstream stages. Like other attributes of a product design, tolerance information changes over time, through successive stages from product planning to detailed design through on-going production. Hence a robust tolerance representation would be mutable and directly related to the design process representation. The incremental refinement of processes and tolerance representations proceeds in symbiotic fashion. Consider, for example, a tooling design/build process. Both lead time and cost for tooling is often highly dependent on the tightness of a tolerance requirement. Scheduling of rough cutting for a die or mold can typically proceed prior to a final tolerance specification, but the finish cut, polishing, etc. must proceed afterward. Conversely, tolerance specification for a snap-fit in a high-precision injection-molded part must be preceded by a decision about assembly 
process (e.g., manual or robotic). For complex assemblies with many parts, the timing and precedence of tolerancing decisions can profoundly affect scheduling and total lead time. Analysis and synthesis for critical tolerance stack-ups is clearly related to process plan refinements. There are opportunities to compress cycle time by improved modeling prior to detailed design, but compatible, incrementally-refined representations of tolerances and processes are the key.

The incremental and continuous, ongoing nature of the process of tolerance decision making enables a natural aggregation/decomposition of tolerancing activities as the design matures. Another way of viewing this is in terms of the pruning that this causes at successive stages in the space of feasible solutions to the design problem. Early on in the design process, the solution space has a staggering cardinality and the tolerancing decisions, if taken in a continuous ongoing fashion, can lead to substantial early reduction in the space of possible solutions thus making the design process efficient. Another alternative view is in terms of marked reduction in design iterations or design rework. In this sense, design for tolerance is similar in spirit to design for manufacturing/assembly [63] that also has the effect of dramatically shrinking the space of solutions and reducing iterations. Furthermore, DFA, DFM, or such other design related strategies may have close coupling with tolerance related decisions and may both influence and be influenced by tolerancing at various stages.

\subsection{Design for Tolerance: A Multilevel Approach}

The first two stages A11 and A12 of the SIMA reference architecture and also the early stages of other assembly design processes (top-down design, Motorola process, Xerox HPD process, and the robust design process) essentially involve mapping customer requirements into product ideas and product specifications. Tolerancing is not directly involved in these early stages, except in very abstract terms; however, these stages provide critical inputs to the tolerancing decisions in the rest of the design process. See Figure 11.

Thus we focus on Stage A13 (Perform Preliminary Design) and Stage A14 (Produce Detailed Designs) of the SIMA reference architecture. We divide these stages into the following four tolerance-related levels (TR Level) and develop a four-level approach to design tolerancing. Note the difference between SIMA stages and tolerance-related levels here.

\section{- SIMA Stage A13: Perform Preliminary Design}

- TR Level 1: Assembly Layout and Configuration

- TR Level 2: Location Logic and Assembly Features

- TR Level 3: Assembly Planning and Sequencing

- SIMA Stage A14: Produce Detailed Designs 
- TR Level 4: Detailed Tolerance Analysis and Synthesis

These levels are fairly representative and generic for electro-mechanical assemblies. Neither the number of levels nor the description of the individual levels is to be viewed as being definitive. Figure 11 captures the essence of this architecture for DFT.

\subsubsection{TR Level 1: Assembly Layout and Configuration}

Once the product concept is known and engineering specifications are generated based on the key characteristics, TR Level 1 of the proposed process can commence. TR Level 1 involves decisions regarding the preliminary assembly layout/configuration. Such decisions may include: rough allocation of space, number of subassemblies, the configuration of critical subassemblies, grouping of components into subassemblies, and rough layout of the assembly. The information available at this level can be described in the form of a liaison diagram (relations between parts or subassemblies), a tree (assembly decomposition), and a partial DFC (to capture whatever location logic is known at this point). Candidate layouts or configurations can be identified and represented using these models. These layouts or configurations and related manufacturing process selection typically might differ in terms of ease of tolerancing. The tolerancing considerations here are at a coarse level and may be directly influenced by customer specifications. To effect such high level tolerancing decisions, aggregate level manufacturing process capability data will be required and is often available at this point. Simple statistical assumptions and probabilistic calculations can be used at this stage. Also, for problems such as manufacturing process selection, optimization formulations such as in Section 4.2.2 can be used.

\subsubsection{TR Level 2: Location Logic and Assembly Features}

At the next level (TR Level 2), the following information is assumed to be available: assembly response functions (approximate); tolerance requirements at interfaces between major subassemblies and parts; and relevant process capability data. The decisions here are concerned with the location logic (how to locate subassemblies and components with respect to one another) and with choosing the appropriate assembly features to go with the location logic. The choice of features itself might depend on the assembly sequence (not the detailed sequence but a precedence specification among major assembly steps). The DFC model is suitable to capture the available/evolving assembly information here. There is close coupling among selection of features, selection of assembly sequence, and creation of DFC. Assembly models such as liaison diagrams are also relevant here. If the assembly is of Type 1, then the assembly features are predominantly decided by the functional requirements; if the assembly is of type 2 , 


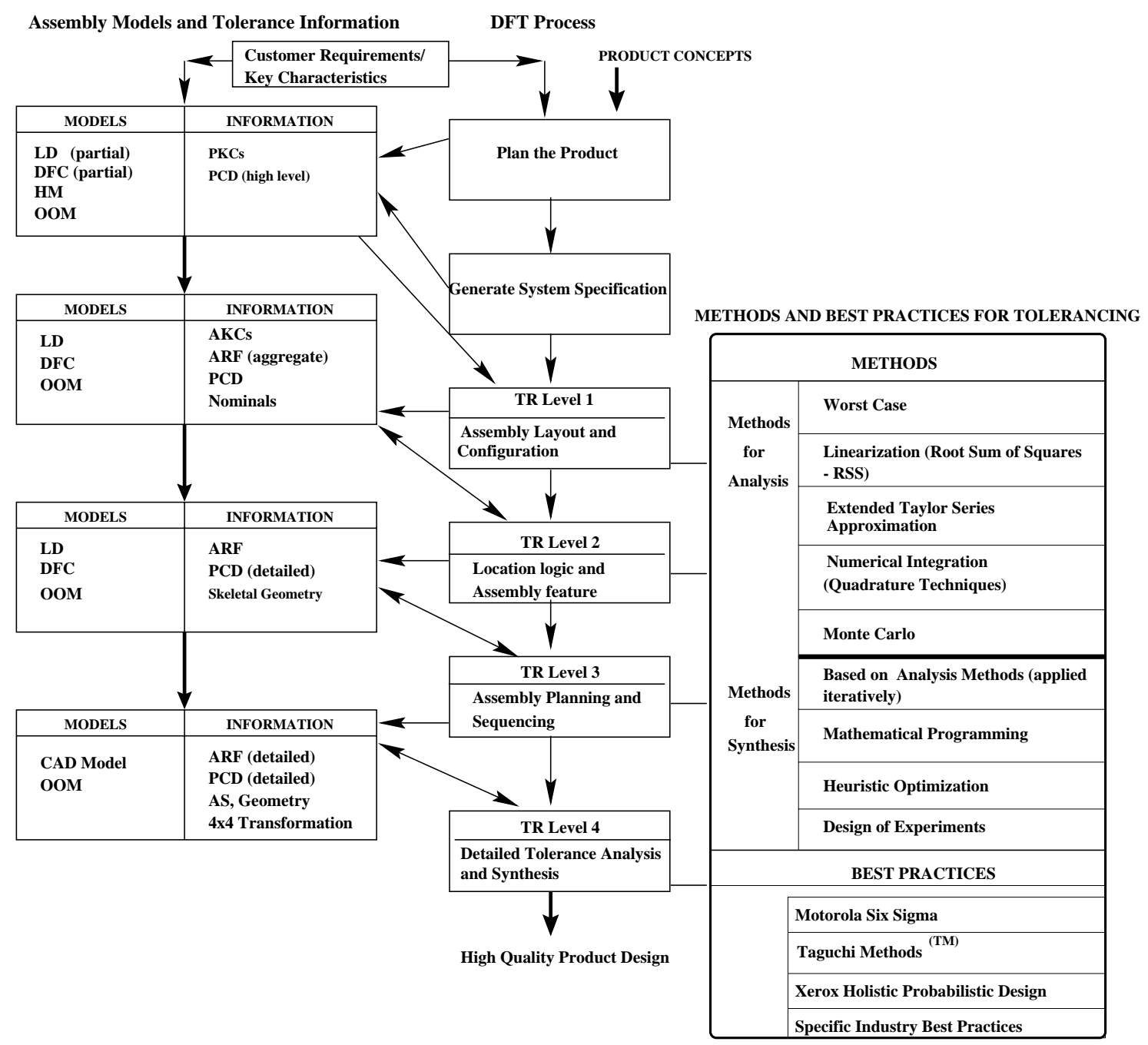

Figure 11: An architecture for design for tolerance. Legend: LD - Liaison Diagram; DFC - Datum Flow Chain; HM - Hierarchical Model; OOM - Object Oriented Model; AS - Assembly Sequence; PKCs - Product Key Characteristics; AKCs - Assembly Key Characteristics; ARF - Assembly Response Function; PCD - Process Capability Data 
then the choice of assembly features is an important problem by itself. In the latter case, the DFC alone will not be adequate to conduct a tolerance analysis. A more detailed model that captures the tolerance flow at this level will be required. Tolerance analysis here can tell us which location logic is better from a tolerancing viewpoint and which set of assembly features would best accomplish tolerance achievement. This stage might also help us to find preliminary target values and tolerances for individual parts.

Statistical tolerance analysis methods listed in Figure 9 are all relevant here. Determining robust nominal values and preliminary settings of tolerances can be accomplished using Taguchi methods or Xerox HPD methodology.

\subsubsection{TR Level 3: Assembly Planning and Sequencing}

We proceed next to TR Level 3 where the detailed assembly response function, detailed process capability data, skeletal geometry of the assembly, assembly features, and, specification of parametric or geometric tolerances of individual parts and features are assumed to be known. From the tolerance specification, one may derive (4 $\mathrm{x}$ 4) matrix transforms for the nominals and variabilities associated with the parts [40]. The decisions here could be with respect to the selection of the detailed assembly sequence that achieves the required tolerance specifications in the best possible way. The models that we employed in the previous stage, like DFC and liaison diagrams, can again be used here. In fact, they are now updated with richer and more detailed information. This kind of representation and analysis is presented in [38], where several data structures to capture tolerance related information are presented. With the information available here, one can also carry out tolerance synthesis.

\subsubsection{TR Level 4: Detailed Tolerance Analysis and Synthesis}

TR Level 4 corresponds to the detailed assembly design stage. Here, the complete assembly sequence is known; geometric data about the parts and features is available; detailed part level tolerance requirements are known; the assembly response function is available in complete form; and low level process capability data is accessible. Detailed tolerance analysis and synthesis can be carried out here. Most tolerancing studies and tolerancing tools available support this level of design.

\subsection{Design for Tolerance: An Integrated Approach}

The multi-level approach to design tolerancing integrates the design process, the assembly models for tolerancing, and the tolerancing methods and best practices. This is captured by Figure 11 . 


\subsubsection{Design Process}

The proposed design process follows the SIMA framework and has four stages: Plan product, generate specifications, perform preliminary design, and produce detailed designs. We have focused on tolerancing decisions during preliminary design and detailed design stages and proposed a four-level approach. It is to be noted that each level above is iterative both internally (feedback within a level) and across (feedback from a given level to a previous level).

The design process delineated here is focused on tolerancing. There are many other subprocesses of the design process that address important issues such as design for assembly, design for manufacturability, design for reliability, etc. All these processes are concurrent, cooperative, and often competitive. A thorough discussion of this is beyond the scope of this paper.

\subsubsection{Assembly Models}

As described in Section 3, there are many assembly modeling approaches that capture the assembly at different stages and at different levels of abstraction. Successive levels of the design for tolerance process will need one or more of these models. The design process evolution is accompanied by a continuous refinement of the assembly models and the tolerancing information.

\subsubsection{Tolerance Analysis and Synthesis}

At successive levels of the DFT process, different kinds of tolerancing decisions need to be taken. These could vary in complexity from simple probabilistic calculations to complex and elaborate computations. As described already, there are a variety of methods and best practices for tolerancing. Which method or best practice to employ at a given level of the DFT process needs careful thought and can depend on a variety of factors such as the product domain, nature of the assembly response function, number of variables involved, and completeness of information.

\section{Example}

Recall the mechanical assembly example of Figure 2. As stated in Section 2, the diagram is conceptual and is not to be viewed as implying any geometry or shape. The conformance or functionality of the assembly is decided by the following criteria:

C1. $Y_{1}=g_{a b}-l_{1} \geq 0$

C2. $Y_{2}=g_{b c}-l_{2} \geq 0$

C3. $Y_{3}=g_{e a} \geq 0$ 
C4. $Y_{4}=g_{c e} \geq 0$

In the above expressions, the tolerance constraints are expressed in terms of linear dimensions. This is because, the gaps and the lengths are 1-D quantities. Therefore the tolerance zone in each case is an interval around the nominal length or nominal gap. More generally, if $g_{a b}$ and $g_{b c}$ represent complex geometrical gap elements, and $R_{a b}$ and $R_{b c}$ represent the tolerance zones for $g_{a b}$ and $g_{b c}$ respectively, the criteria C1 and $\mathbf{C} \mathbf{2}$ above can be expressed as:

$$
\begin{aligned}
& g_{a b} \in R_{a b} \\
& g_{b c} \in R_{b c}
\end{aligned}
$$

The tolerance zones $R_{a b}$ and $R_{b c}$ will have the lengths $l_{1}$ and $l_{2}$, respectively, among their parameters. For the sake of simplicity, we shall consider here only parametric tolerances. Consequently, the tolerance zones become intervals. The discussion is similar for geometric tolerances, with appropriate extensions and reinterpretation.

We now discuss how tolerance related decisions can be taken at the four levels of the design for tolerance process (Figure 11).

\subsection{Selecting a Configuration}

Figure 12 shows three possible ways of configuring the five parts A, B, C, D, and E as product P; there could be other configurations as well. In Configuration 1, all five parts are treated as individual components and the assembly takes place in stages. In Configuration 2, P comprises E, D, and a subassembly that consists of components $\mathrm{A}, \mathrm{B}$, and $\mathrm{C}$. The motivation for considering this configuration might be that the subassembly is available off-the-shelf from a known vendor. Likewise, Configuration 3 is another candidate. In this case, the subassembly comprising A and B might be available from a different vendor. It is clear that the process capabilities and the associated parametric variations of the components and subassemblies will influence the choice of the configuration.

To decide which of the above three configurations is best from a tolerancing viewpoint, we need to determine how well the criteria $\mathbf{C 1}, \mathbf{C 2}, \mathbf{C} 3$, and $\mathbf{C 4}$ are met by the configurations. A natural way of doing this is to compute the probabilities:

$$
\begin{aligned}
& p_{1}=\operatorname{Pr}\left\{g_{a b} \geq l_{1}\right\} \\
& p_{2}=\operatorname{Pr}\left\{g_{b c} \geq l_{2}\right\} \\
& p_{3}=\operatorname{Pr}\left\{g_{e a} \geq 0\right\} \\
& p_{4}=\operatorname{Pr}\left\{g_{c e} \geq 0\right\}
\end{aligned}
$$

The following data is known about these configurations: 

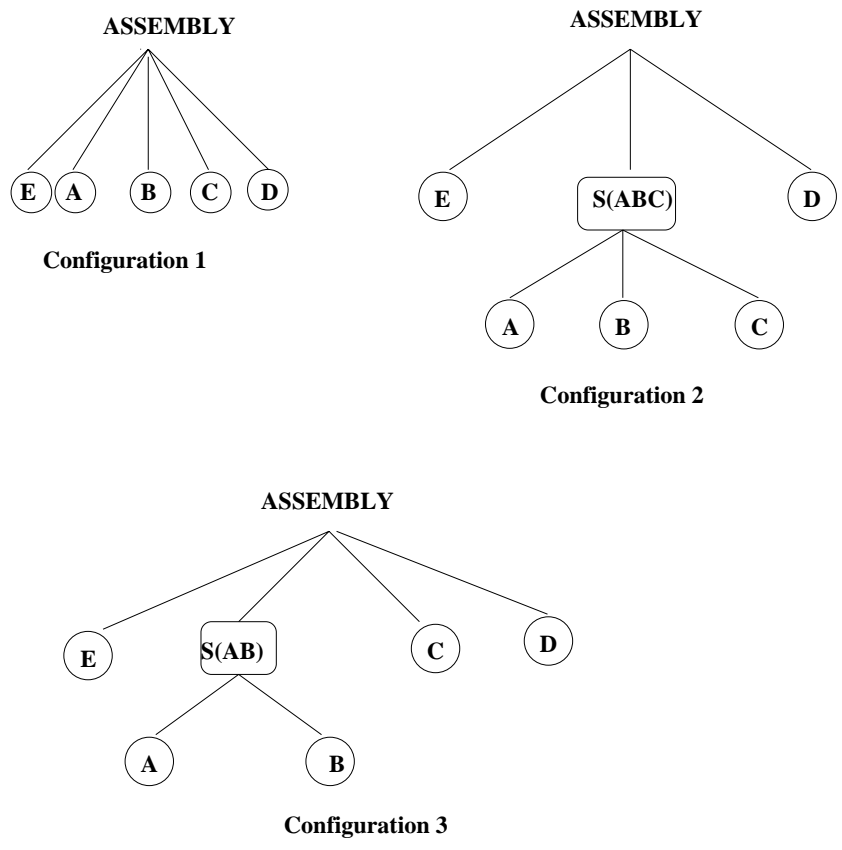

Figure 12: Three different configurations of the assembly

- In the case of Configuration 1 , the random variables $l_{a}, l_{b}, l_{c}, l_{e}, l_{1}$, and $l_{2}$ are known (available from the vendors supplying these components or from local factory data). This means we know either the probability distribution or at the least the first few moments of each random variable. The gaps $g_{a b}$ and $g_{b c}$ are not known since they depend on the assembly process. Similarly, the gaps $g_{e a}$ and $g_{c e}$ are also not known since they also depend on the assembly process. In fact, $g_{e a}$ and $g_{c e}$ are related by the following equation:

$$
l_{e}=g_{e a}+l_{a}+g_{a b}+l_{b}+g_{b c}+l_{c}+g_{c e}
$$

If the assembly sequence is such that $g_{e a}$ is decided first (that is, A is assembled to E earlier than C), the above equation can be used to determine $g_{c e}$ (provided $g_{a b}$ and $g_{b c}$ are known). On the other hand, if $g_{c e}$ is decided first, $g_{a e}$ can be determined using the above equation.

- In respect of Configuration 2, the following are known: The lengths $l_{a}, l_{b}, l_{c}, l_{e}, l_{1}$, $l_{2}$, and the gaps $g_{a b}$ and $g_{b c}$. The gaps $g_{e a}$ and $g_{c e}$ depend on the assembly process. Since $l_{1}$ and $g_{a b}$ are known, the probability $p_{1}$ can be computed. Similarly, the probability $p_{2}$ can be computed since $l_{2}$ and $g_{b c}$ are known.

- In the case of Configuration 3, all the length-related random variables are known, while among the gaps only $g_{a b}$ is known. Thus we can compute $p_{1}$ but not $p_{2}$. 
The key to selecting the best among these configurations lies in choosing an important subset of criteria (probabilities) on which to base the decision, and then the ability to compute the chosen probabilities without bringing in assembly sequence or other downstream concerns. In the present case, it is reasonable to base the decision on $p_{1}$ and $p_{2}$, ignoring $p_{3}$ and $p_{4}$. To compute $p_{1}$ and $p_{2}$ for the above three configurations, we proceed as follows. It is straightforward in the case of Configuration 2, as already explained. In respect of Configuration $3, p_{1}$ can be computed easily as explained above. To compute $p_{2}, g_{b c}$ can be assumed to be the same as for Configuration 2 (this makes the comparison fair). As for Configuration 1, $g_{b c}$ can be assumed to be the same as in Configurations 2 and $3 ; g_{a b}$ can be assumed to be either the minimum of the values of this gap for Configurations 2 and 3 (optimistic) or maximum of the values (pessimistic).

Having chosen a particular configuration (say configuration 1), another important decision needs to be taken. This concerns the supplier selection or manufacturing process selection. If the components A, B, C, D, and E are being supplied by two separate vendors and the components have differing specifications and costs, then which supplier to choose is an important question that can again be partially resolved by computing the probabilities above. Here, cost considerations also become important. If there is a wider choice of suppliers and each supplier has multiple offerings, the problem can be resolved by design of experiments or Taguchi methods, with a carefully chosen cost function. Another important decision concerns the manufacturing process selection. Here, the problem is to choose the best combination of manufacturing or assembly processes to make the components and assemble them, so as to satisfy tolerance requirements and minimize manufacturing/assembly cost. The optimization formulation shown in Section 4.2.2 addresses this type of synthesis problem.

\subsection{Selecting Location Logic and Assembly Features}

In this stage of design, our interest is in fixing the location logic, which often allows the choice of assembly features. Figure 12 shows four candidate DFCs; there could be other candidates as well. In Candidate 1, A and $\mathrm{C}$ in some order are first assembled into $\mathrm{E}$ and then $\mathrm{B}$ is located with respect to $\mathrm{A}$ and $\mathrm{C}$. Next, D is assembled with respect to $\mathrm{A}, \mathrm{B}$, and $\mathrm{C}$ to yield the proper gaps. In Candidate 2 logic, $\mathrm{B}$ is the first one to be assembled into the envelope, followed by $\mathrm{A}$ and $\mathrm{C}$ in some order and thereafter, $\mathrm{D}$ is assembled. Candidate 3 assemblies correspond to those sequences in which $\mathrm{B}$ is assembled in the middle between $\mathrm{A}$ and $\mathrm{C}$ (these two in any order). Note that $\mathrm{D}$ is assembled last in candidate logics 1,2 , and 3 . In candidate 4 , a fixture $\mathrm{F}$ can possibly be used to hold $\mathrm{D}$ and then $\mathrm{A}, \mathrm{B}$, and $\mathrm{C}$ are properly located with reference to the position of D. E is finally assembled to hold A, B, C, and D. The use of a fixture is motivated by higher positioning accuracies that can possibly be achieved with welldesigned fixtures. From the conceptual diagram of Figure 2, one can also visualize how a particular location logic can influence the nature and choice of mating features. 


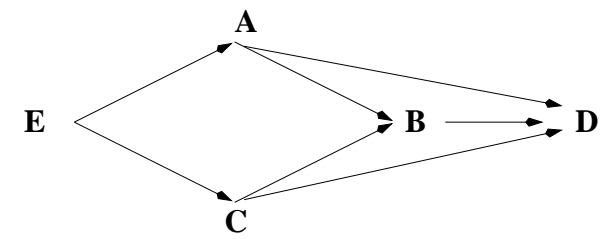

Candidate 1

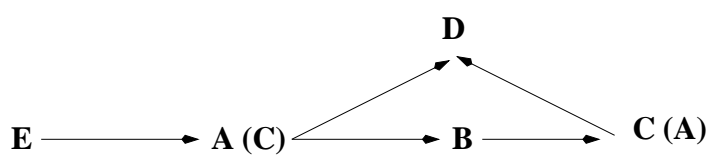

Candidate 3

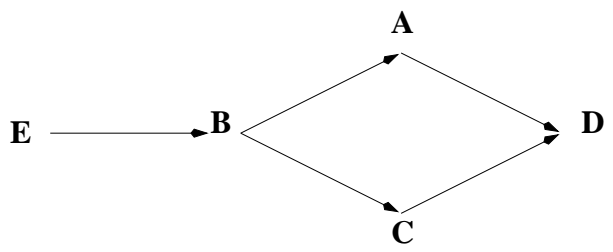

Candidate 2

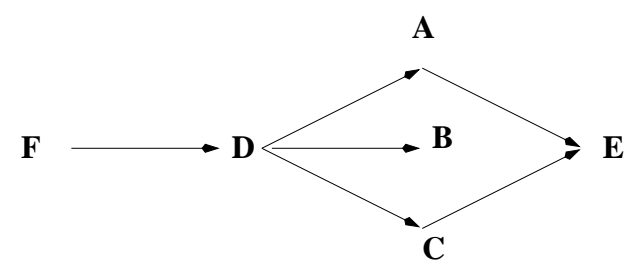

Candidate 4

Figure 13: Datum flow chains representing different location logics

To compare the above four candidates, we need to compute the probabilities $p_{1}, p_{2}$, $p_{3}$, and $p_{4}$. Recall that we know the distributions of $l_{a}, l_{b}, l_{c}, l_{1}, l_{2}$, and $l_{e}$. The distributions or moments of the gaps are now to be computed knowing the location logic and relevant process capability data. For instance, consider candidate 1.

- Since A and C are first assembled into E, the distribution or moments of $g_{e a}$ and $g_{c e}$ can be computed first (assumed to be assembled first). The probabilities $p_{3}$ and $p_{4}$ can then be computed. These computations will need process capability data about the assembly operations.

- Next, B is placed inside the envelope. Knowing the process capability data for this operation, we can compute the distributions or moments of $g_{a b}$ and $g_{b c}$.

- Finally, knowing the process capability of assembling $\mathrm{D}$, the probabilities $p_{1}$ and $p_{2}$ can be computed.

We may remark that Candidate 2 is likely to be the best since it enables variation to be transferred to where it is not important. On the other hand, if there is high variability in the dimension of $\mathrm{B}$, then Candidate 3 may turn out to be a better choice. Also, note that design for assembly considerations may negate the choice of Candidate 1 for the reason that assembly may be difficult to achieve since component $B$ is to be juxtaposed between $\mathrm{A}$ and $\mathrm{C}$, providing for the desired gaps. 
Similar Statistical computations can be carried out using the tolerance analysis methods of Section 4.1. Best practices, such as the Motorola six sigma program and the Xerox HPD, are also suitable for such computations.

If the parts are 3-D, then instead of linear dimensions as above, more general methods will have to be used. If $(4 \times 4)$ matrix representation is available for the parts and their tolerances, then the matrix transforms can be used in the computations [40].

\subsection{Selection of Assembly Sequence}

Each candidate logic can correspond to multiple assembly sequences, thus selecting a sequence occurs later than selecting a location logic. We observed in the previous subsection that Candidate 3 is likely to be better if there is high uncertainty involved in the dimension of $\mathrm{B}$. If this candidate is chosen, then there are two possible sequences: $\mathrm{E} \rightarrow \mathrm{A} \rightarrow \mathrm{B} \rightarrow \mathrm{C} \rightarrow \mathrm{D}$ or $\mathrm{E} \rightarrow \mathrm{C} \rightarrow \mathrm{B} \rightarrow \mathrm{A} \rightarrow \mathrm{D}$. If this assembly were of Type 1, then, as observed previously, (in Section 2), both these sequences will result in the same tolerance chain and hence the same values for the probabilities $p_{i}(i=1,2,3,4)$. However, if we regard this as a Type 2 assembly (that is, features are formed during the assembly process with the use of fixtures), then the two sequences could differ with respect to tolerance achievement. Using the data available about the distribution of the lengths, and process capabilities for the individual parts, one can compute the probabilities $p_{1}, p_{2}, p_{3}$, and $p_{4}$. Then decide which sequence is better. For example, if $l_{a}$ has more variability than $l_{c}$, then the second sequence is likely to be better than the first sequence, since the higher variation of $l_{a}$ can be transferred to where it is not important. In this case, this is intuitively clear but in complex assemblies, one necessarily needs to carry out such analysis.

\subsection{Detailed Analysis and Synthesis}

When the design process reaches advanced stages, tolerance analysis and synthesis can be done in a comprehensive way since we have access to to detailed data.

\subsubsection{Tolerance Analysis}

For example, the following information may be known:

- Assembly sequence: Say, $\mathrm{E} \rightarrow \mathrm{B} \rightarrow \mathrm{A} \rightarrow \mathrm{C} \rightarrow \mathrm{D}$

- Distributions of $l_{a}, l_{b}, l_{c}, l_{e}, l_{1}$, and $l_{2}$, or alternatively their nominals $N_{a}, N_{b}, N_{c}$, $N_{e}, N_{1}$, and $N_{2}$; and corresponding tolerances $T_{a}, T_{b}, T_{c}, T_{e}, T_{1}$, and $T_{2}$.

- Process capabilities of different assembly steps in the assembly sequence 
First, B and A are located on the envelope, leaving the right amount of gap $g_{a b}$. Knowing the $C_{p}$ and $C_{p k}$ of this step, the probability $p_{1}$ can be computed. Also, it is easy to see that $p_{3}=1$. The next operation is to locate and place the component $\mathrm{C}$ so as to get the correct gap between $\mathrm{B}$ and $\mathrm{C}$ and also avoid interference between $\mathrm{C}$ and E. One can then compute the probabilities $p_{2}$ and $p_{4}$, knowing the appropriate process capability data.

Here again, either statistical tolerance analysis methods could be used. Very detailed analysis can be done using Monte Carlo simulation.

The discussion above has again assumed linear dimensions and tolerances. If the geometry of the individual components and the assembly are known, then one can specify the data in terms of the ANSI standard on geometric tolerances and use $(4 \mathrm{x}$ 4) matrix transforms and repeat the above computations.

\subsubsection{Design}

Design or synthesis can assume several forms, see for example, Harry and Stewart [50]. The possibilities include: optimization of nominal dimensions; optimization of tolerances; and optimal allocation of overall assembly variation across individual parts.

Let us say the desired probability of non-conformance is $3.4 \mathrm{ppm}$, as in the Motorola six sigma program. If $\mathrm{A}, \mathrm{B}, \mathrm{C}$, and D are from external vendors and all appropriate data is known (nominals and either tolerances or standard deviations) for those, then for a given tolerance $T_{e}$ of the envelope, one can determine the nominal value $N_{e}$ so as to assure the required probability of conformance. This can be done both optimistically (no shifts in the process mean) and realistically (in the presence of shifts in the process mean).

Using Taguchi methods or Xerox HPD, one can determine robust nominals for all the parts involved, that is, the combination of nominals of the individual parts for which the effect of variations is minimized.

On the other hand, if all relevant data for A, B, C, and D is known, and the nominal $N_{e}$ of the envelope is fixed, one can determine the tolerance $T_{e}$ of the envelope so as to achieve a probability of non-conformance of say, $3.4 \mathrm{ppm}$. Here we are determining the capability of the process that fabricates the envelope.

\section{Discussion and Future Work}

In this paper, we have outlined a continuous, multi-level approach to design tolerancing of electro-mechanical assemblies. The architecture integrates three main elements: Assembly models for tolerancing; methods and best practices for tolerancing; and the evolving design process. We have delineated a four level approach for incremental 
design tolerancing and illustrated the methodology for a simple, representative, mechanical assembly. The discussion has centered on parametric or linear tolerances. Extension to functional tolerancing is straightforward since the analysis and synthesis methods can handle arbitrary, possibly nonlinear, functional relationship between the individual piece-part characteristics and the assembly response. Also, by suitably defining tolerance zones, extension to geometrical tolerances is possible. Since the ultimate test of any such methodology is in successful application to industry-level products, a logical next step would be to look into industry-level implementation of the proposed approach. There are two important directions for further work on this topic. These are: implementation of a DFT environment and facilitation of standards development.

\subsection{Implementation of a DFT Environment}

As Figure 11 suggests, computer implementation of an automated design for tolerance environment will involve integrating together the assembly models and the tolerancing techniques with the design process. Tolerance analysis and tolerance synthesis during the assembly design stage affect the design process in an influential way and therefore lead to a better understanding and formulation of the design process. Since improvements to the design process require effective modeling of the process, the proposed work will offer valuable insights into process modeling. For example, as tolerancerelated information becomes available in increasing detail during the design process, both the process and product representations undergo successive refinements. This needs to be captured by the model. A number of methods have been proposed over the years to model design processes. However, these methods have several inadequacies. An ideal process model should enable faithful modeling of precedence relations, constraints, iterations, side effects, dependencies, abstraction, cost factors, and timeto-market determinants $[64,65]$. The proposed work will help understand the process modeling requirements for assembly design. The work also raises interesting issues such as finding an integrated representation formalism for assembly modeling and also design process modeling. As already stated in Section 3.3, object oriented models can form the foundation of such integrated product-process models.

\subsection{Standards Development}

It is expected that the proposed work on assembly modeling and assembly representation will provide preliminary specifications that can serve as the basis for assembly standards. The current standard (AP203) only allows the representation of an assembly as a collection of $3 \mathrm{D}$ objects positioned and oriented in space. It does not make any provision for the capture of logical relationships between parts, mating feature relationships, part functionality, kinematic degrees of freedom, and tolerance information. The work here will provide useful inputs to the development of such a standard. 
Dimensional tolerancing has mostly evolved as an industrial practice without strong theoretical foundations [16]. The best tolerancing practices were collected and made available through an evolving series of tolerancing standards [24-27]. All international, and most national, standards have codified only classical tolerancing [7]. The DIN (Deutsches Institut für Normung- German Institute for Standardization) standard issued in Germany [66] was a serious attempt at standardizing statistical tolerancing. The latest ASME Y14.5M-1994 standard on dimensioning and tolerances [26] provides a provision for including statistical tolerances. Currently, mathematical definitions of the syntax and semantics of statistical tolerancing are under development for inclusion into standards. An ISO standard for statistical tolerancing is evolving [7]. Improved understanding of the assembly design process from a tolerancing viewpoint and integration of various best practices at various stages of this design process will no doubt provide a critical input to the formulation of tolerancing representation and standards.

\section{Acknowledgments}

This work is sponsored by the SIMA (Systems Integration for Manufacturing Applications) program at the National Institute of Standards and Technology and the RaDEO (Rapid Design Exploration and Optimization) program at the Defense Advanced Research Projects Agency. We would like to acknowledge the value of many discussions with Dr Jean Parks from the Xerox Corporation, Webster, and with Dr. Raghu Kacker of the Statistical Engineering Division at NIST.

\section{Disclaimer}

No approval or endorsement of any commercial product by the National Institute of Standards and Technology is intended or implied. Certain commercial equipments, instruments, or materials are identified in this report in order to facilitate better understanding. Such identification does not imply recommendations or endorsement by the National Institute of Standards and Technology, nor does it imply the materials or equipment identified are necessarily the best available for the purpose.

\section{References}

[1] R. Sudarsan, K. Lyons, and M. Pratt (Editors). Assembly Level Tolerancing: Standards and Implementation Issues. Workshop Handout, National Institute of Standards and Technology, 1997.

[2] K.W. Lyons, S. Angster, P. Hart, Y. Narahari, V. Rajan, and R. Sudarsan. OpenADE: Open Assembly Design Environment. Technical report, National Institute of Standards and Technology, 1997 (In preparation). 
[3] D. J. Lee and A.C. Thornton. Enhanced key characteristics identification methodology for agile design. In Proceedings of the Agile Manufacturing Forum, Boston, MA, March 1996.

[4] T. Cunningham, R. Mantripragada, D.Lee, A. Thornton, and D. Whitney. Definition, analysis, and planning of a flexible assembly process. In Proceedings of the Japan-USA Symposium on Flexible Automation, Boston, USA, pages 767-778, 1996.

[5] R. Mantripragada, T.W. Cunningham, and D.E. Whitney. Assembly oriented design: A new approach to designing assemblies. In M.J. Pratt, R.D. Sriram, and M.J. Wozny, editors, Product Modeling for Computer Integrated Design and Manufacture, pages 308324. Chapman \& Hall, 1997.

[6] D.E. Whitney. The potential for assembly modeling in product development and manufacturing. Technical report, The Center for Technology, Policy, and Industrial Development, MIT, Cambridge, MA, USA, 1996.

[7] V. Srinivasan and M.A. O'Connor. Towards an ISO standard for statistical tolerancing. In F. Kimura, editor, Proceedings of the Fourth CIRP Design Seminar on ComputerAided Tolerancing, pages 159-172. Chapman \& Hall, London, 1996.

[8] U. Roy and Bing Li. Representation and intrepretation of geometric tolerances for polyhedral objects, Part I:Form Tolerances. CAD, 30(2):151-161, February 1998.

[9] U. Roy and Bing Li. Representation and intrepretation of geometric tolerances for polyhedral objects, Part II:Size, Orientation, and Positional Tolerances. Submitted for Publication, 1998.

[10] U.Roy and Ying che Fang. Tolerance representation scheme for a 3-D product in object oriented programming environment. IIE Transaction on Design and Manufacturing, 28:809-819, 1996.

[11] U.Roy, C.R.Liu, and T.C.Woo. A review of dimensioning and toleraning:Representation and Processing. CAD, 23(7):466-483, September 1991.

[12] D.H. Evans. Statistical tolerancing: The state of the art, Part I. background. Journal of Quality Technology, 6:188-195, 1974.

[13] D.H. Evans. Statistical tolerancing: The state of the art, Part II. Method for estimating moments. Journal of Quality Technology, 7:1-12, 1975.

[14] D.H. Evans. Statistical tolerancing: The state of the art, Part III. Shifts and drifts. Journal of Quality and Technology, 7(2):72-76, 1975.

[15] K.W. Chase and A. Parkinson. A survey of research in the application of tolerance analysis to the design of mechanical assemblies. Research in Engineering Design, 1(3):2337, 1991. 
[16] H. Voelcker. A current perspective on tolerancing and metrology. Manufacturing Review, 6(4):258-268, 1993.

[17] J.U. Turner. Current tolerancing packages. In V. Srinivasan and H. Voelcker, editors, Proceedings of the 1993 International Forum on Dimensional Tolerancing and Metrology. ASME, New York, 1993.

[18] S.D. Nigam and J.U. Turner. Review of statistical approaches to tolerance analysis. Computer Aided Design, 27(1):6-15, January 1995.

[19] A. Kusiak and C.X. Feng. Deterministic tolerance synthesis: A comparative study. Computer Aided Design, 27(10):759-768, October 1995.

[20] M.J. Harry. The nature of six sigma quality. Technical report, Government Electronics Group, Motorola inc., Scottsdale, Arizona, 1987.

[21] R.N. Kacker. Taguchi methods. In Handbook of Statistical Methods for Engineers, pages 19.1-19.27. Mc-Graw Hill, 1988.

[22] J.M. Parks. Holistic approach and advanced techniques and tools for tolerance analysis and synthesis. In F. Kimura, editor, Computer-Aided Tolerancing, pages 251-269. Chapman and Hall, London, 1996.

[23] J.M. Parks. Advanced methodology and software for tolerancing and stochastic optimization. In Proceedings of the Fifth International Seminar on Computer-Aided Tolerancing, Toronto, Canada, April 1997.

[24] American National Standards Institute. Dimensioning and Tolerancing: ANSI Y 14.5M1982. American Society of Mechanical Engineers, New York, 1983.

[25] ASME. Dimensioning and Tolerancing. American Society of Mechanical Engineers, New York, 1983.

[26] American Society of Mechanical Engineers. ASME Y 14.5M-1994: Dimensioning and Tolerancing. ASME, New York, 1994.

[27] R.K. Walker and V. Srinivasan. Creation and evolution of the ASME Y14.5.1 standard. Manufacturing Review, 7(1):16-23, 1994.

[28] International Business Machines Inc. Statistical Dimensioning: IBM Corporate Standard 0-0-1017-0. IBM, USA, 1960.

[29] A.G. Neumann. The new ASME Y14.5M standard on dimensioning and tolerancing. Manufacturing Review, 7(1):9-15, 1994.

[30] V. Tipnis (Editor). Selected Case Studies in the Use of Tolerance and Deviation Information During Design of Representative Industrial Products. ASME Press, New York, USA, 1992. 
[31] R.E. Altschul and F.W. Scholz. Case study in statistical tolerancing. Manufacturing Review, 7(1):52-56, March 1994.

[32] S. Srikanth and J.U. Turner. Toward a unified representation of mechanical assembly. Engineering with Computers, 6(2):103-112, 1990.

[33] J.R. Dixon and M.R. Duffey. A program of research in mechanical design: Computerbased models and representations. Mechanism and Machine Theory, 25(3), 1990.

[34] M. Mantyla. A modeling system for top-down design of assembled products. IBM Journal of Research and Development, 34(5):636-659, 1990.

[35] M.A. Wesley, T. Lozano-Perez, L.I. Lieberman, M.A. Lavin, and D.D. Grossman. A geometric modeling system for automated mechanical assembly. IBM Journal of Research and Development, 24(1):64-74, 1980.

[36] K. Lee and D.C. Gossard. A hierarchical data structure for representing assemblies: Part 1. Computer Aided Design, 17(1):15-19, 1985.

[37] D.C. Gossard, R.P. Zuffante, and H. Sakurai. Representing dimensions, tolerances, and features in MCAE systems. IEEE Computer Graphics ana Applications, 8(2):51-59, 1988.

[38] N. Wang and T.M. Ozsoy. Representation of assemblies for automatic tolerance chain generation. Engineering with Computers, 6(2):121-126, 1990.

[39] S. Callahan and J. Heisserman. A product representation to support process automation. In M.J. Pratt, R.D. Sriram, and M.J. Wozny, editors, Product Modeling for Computer Integrated Design and Manufacture, pages 285-296. Chapman \& Hall, 1997.

[40] D.E. Whitney, O.L. Gilbert, and M. Jastrzebski. Representation of geometric variations using matrix transforms for statistical tolerance analysis in assemblies. Research in Engineering Design, 6:191-210, 1994.

[41] U. Roy and C.R.Liu. Establishment of functional relationships between product components in assembly database. CAD, 20(10):570-580, December 1988.

[42] U. Roy, P.Banerjee, and C.R.Liu. Design of an automated assembly environment. CAD, 21(9):561-569, November 1989.

[43] N.P. Juster. Modeling and representation of dimensions and tolerances: A survey. Computer Aided Design, 24(1):3-17, 1992.

[44] J.U. Turner. Relative positioning of parts in assemblies using mathematical programming. Computer Aided Design, 22(7):394-400, September 1990.

[45] S.R. Gorti, A. Gupta, , G.J. Kim, R.D. Sriram, and A. Wong. An object-oriented representation for product and design processes. Technical report, National Institute of Standards and Technology, Gaithersburg, Maryland, 1997. 
[46] S.R. Gorti and R.D. Sriram. From symbol to form: A framework for conceptual design. Computer Aided Design, 28(11):853-870, 1996.

[47] A. Wong and R.D. Sriram. SHARED: An information model for cooperative product development. Research in Engineering Design, 5:21-39, 1993.

[48] J.K. Gui and Mantyla. Functional understanding of assembly modeling. Computer Aided Design, 26(6):435-451, June 1994.

[49] O. Bjorke. Computer Aided Tolerancing. New York: ASME Press, Second Edition, 1989.

[50] M.J. Harry and R. Stewart. Six sigma mechanical design tolerancing. Technical report, Government Electronics Group, Motorola inc., Scottsdale, Arizona, 1988.

[51] M.J. Harry and J.R. Lawson. Six Sigma Producibility Analysis and Process Characterization. Motorola University Press, 1991.

[52] J.M. Parks. The unified methodology on design for latitude as a concept and capability for tolerance analysis and synthesis. In V.J. Tipnis, editor, Selected Case Studies in the Use of Tolerance and Deviation Information during Design of Representative Industrial Products, pages 129-150. ASME, New York, 1992.

[53] J.M. Parks. The two enablers for holistic probabilistic design: Methodology and a complete suite of tools for stochastic analysis. In Proceedings of the Third International Conference on Stochastic Structural Dynamics, 1995.

[54] R.N. Kacker. Off-line quality control, parameter design, and the taguchi method. Journal of Quality and Technology, 17(4):176-188, 1985.

[55] M.S. Phadke. Quality Engineering using Robust Design. Prentice Hall, Englewood Cliffs, NJ, USA, 1989.

[56] J. Lee, T.C. Woo, and S.Y. Chou. Tolerance synthesis for non-linear systems based on non-linear programming. IIE Transactions, 25(1):51-61, 1993.

[57] M.P. Iannuzzi and E. Sandgren. Tolerance optimization using genetic algorithms: Benchmarking with manual analysis. In F. Kimura, editor, Proceedings of the Fourth CIRP Design Seminar on Computer-Aided Tolerancing, pages 220-234. Chapman \& Hall, London, 1996.

[58] S. Kanai, M. Onozuka, and H. Takahashi. Optimal tolerance synthesis by genetic algorithm under the machining and assembling constraints. In F. Kimura, editor, Proceedings of the Fourth CIRP Design Seminar on Computer-Aided Tolerancing, pages 235-250. Chapman \& Hall, London, 1996.

[59] J. Lee and G.E. Johnson. Optimal tolerance allotment using a genetic algorithm and truncated Monte Carlo simulation. Computer Aided Design, 25(9):601-611, 1993. 
[60] S. Kotz and N.L. Johnson. Process Capability Indices. Chapman and Hall, London, 1993.

[61] A. Bender. Benderizing tolerances - a simple probability method of handling tolerances for limit stack-ups. Graphic Science, pages 17-23, DEcember 1962.

[62] E.J. Barkmeyer (Editor). SIMA (Systems Integration for Manufacturing Applications) reference architecture, Part 1: Activity models. Technical report, National Institute of Standards and Technology, Internal Report 5939, December 1996.

[63] G. Boothroyd. Product design for manufacturing and assembly. Computer Aided Design, 26(7):505-520, July 1994.

[64] M.R. Duffey and J.R. Dixon. Managing the product realization process: A model for aggregate cost anf time-to-market evaluation. Concurrent Engineering: Research and Applications, 1(1), 1993.

[65] K.W. Lyons and M.R. Duffey. Requirements, methods, and research issues for modeling the product realization process. In J. Brown and D. O'Sullivan, editors, Re-engineering the Enterprise. Chapman \& Hall, 1996.

[66] DIN 7186 Baltt 1. Statistiche Tolerierung: Begriffe, Anwendungsrichtlinien und Zeichnungsangaben. Deutsche Normen, Berlin, BRD, 1974. 